\title{
The evolution of the luminosity functions in the FORS deep field from low to high redshift
}

\section{The red bands $\star$}

\author{
A. Gabasch ${ }^{1,2}$, U. Hopp ${ }^{1,2}$, G. Feulner ${ }^{1,2}$, R. Bender ${ }^{1,2}$, S. Seitz ${ }^{1}$, R. P. Saglia ${ }^{2}$, J. Snigula ${ }^{1,2}$, \\ N. Drory ${ }^{3}$, I. Appenzeller ${ }^{4}$, J. Heidt ${ }^{4}$, D. Mehlert ${ }^{4}$, S. Noll ${ }^{2,4}$, A. Böhm ${ }^{5}$, K. Jäger ${ }^{5}$, and B. Ziegler ${ }^{5}$ \\ 1 Universitäts-Sternwarte München, Scheinerstr. 1, 81679 München, Germany \\ e-mail: gabasch@usm.uni-muenchen.de \\ 2 Max-Planck-Institut für Extraterrestrische Physik, Giessenbachstraße, 85748 Garching, Germany \\ 3 McDonald Observatory, University of Texas at Austin, Austin, Texas 78712, USA \\ ${ }^{4}$ Landessternwarte Heidelberg, Königstuhl, 69117 Heidelberg, Germany \\ 5 Institut für Astrophysik, Friedrich-Hund-Platz 1, 37077 Göttingen, Germany
}

Received 4 August 2005 / Accepted 11 October 2005

\section{ABSTRACT}

We present the redshift evolution of the restframe galaxy luminosity function (LF) in the red $r^{\prime}, i^{\prime}$, and $z^{\prime}$ bands, as derived from the FORS Deep Field (FDF), thus extending our earlier results to longer wavelengths. Using the deep and homogeneous $I$-band selected dataset of the FDF, we were able to follow the red LFs over the redshift range $0.5<z<3.5$. The results are based on photometric redshifts for 5558 galaxies derived from the photometry in 9 filters and achieving an accuracy of $\Delta z /\left(z_{\text {spec }}+1\right) \leq 0.03$ with only $\sim 1 \%$ outliers. A comparison with results from the literature shows the reliability of the derived LFs. Because of the depth of the FDF, we can give relatively tight constraints on the faint-end slope $\alpha$ of the LF; the faint-end of the red LFs does not show a large redshift evolution and is compatible within $1 \sigma$ to $2 \sigma$ with a constant slope over the redshift range $0.5 \lesssim z \lesssim 2.0$. Moreover, the slopes in $r^{\prime}, i^{\prime}$, and $z^{\prime}$ are very similar to a best-fitting value of $\alpha=-1.33 \pm 0.03$ for the combined bands. There is a clear trend of $\alpha$ to steepen with increasing wavelength: $\alpha_{\mathrm{UV} \& u^{\prime}}=-1.07 \pm 0.04 \rightarrow \alpha_{g^{\prime} \& B}=-1.25 \pm 0.03 \rightarrow$ $\alpha_{r^{\prime} \& i^{\prime} \& z^{\prime}}=-1.33 \pm 0.03$. We subdivided our galaxy sample into four SED types and determined the contribution of a typical SED type to the overall LF. We show that the wavelength dependence of the LF slope can be explained by the relative contribution of different SED-type LFs to the overall LF, as different SED types dominate the LF in the blue and red bands. Furthermore we also derived and analyzed the luminosity density evolution of the different SED types up to $z \sim 2$.

We investigated the evolution of $M^{*}$ and $\phi^{*}$ by means of the redshift parametrization $M^{*}(z)=M_{0}^{*}+a \ln (1+z)$ and $\phi^{*}(z)=\phi_{0}^{*}(1+z)^{b}$. Based on the FDF data, we found only a mild brightening of $M^{*}\left(a_{r^{\prime}} \sim-0.8\right.$, and $\left.a_{i^{\prime}, z^{\prime}} \sim-0.4\right)$ and a decreasing $\phi^{*}\left(b_{r^{\prime}, i^{\prime}, z^{\prime}} \sim-0.6\right)$ with increasing redshift. Therefore, from $\langle z\rangle \sim 0.5$ to $\langle z\rangle \sim 3$ the characteristic luminosity increases by $\sim 0.8, \sim 0.4$, and $\sim 0.4$ mag in the $r^{\prime}, i^{\prime}$, and $z^{\prime}$ bands, respectively. Simultaneously the characteristic density decreases by about $40 \%$ in all analyzed wavebands. A comparison of the LFs with semianalytical galaxy formation models by Kauffmann et al. (1999) shows a similar result to the blue bands: the semi-analytical models predict LFs that describe the data at low redshift very well, but show growing disagreement with increasing redshifts.

Key words. galaxies: luminosity function, mass function - galaxies: fundamental parameters - galaxies: high-redshift galaxies: distances and redshifts - galaxies: evolution

\section{Introduction}

One major effort in extragalactic astronomy has been to derive and analyze the restframe galaxy luminosity function in different bandpasses and redshift slices, in order to follow the time

* Based on observations collected with the VLT on Cerro Paranal (Chile) and the NTT on La Silla (Chile) operated by the European Southern Observatory in the course of the observing proposals 63.O-0005, 64.O-0149, 64.O-0158, 64.O-0229, 64.P-0150, 65.O-0048, 65.O-0049, 66.A-0547, 68.A-0013, and 69.A-0014. evolution of galaxy populations by a statistical approach. This is of particular importance because the energy output at different wavelengths is dominated by stars of different masses. While galaxy luminosities measured in the ultraviolet are sensitive to the energy output of hot, short-living $\mathrm{O}$ and $\mathrm{B}$ type stars and, therefore, to ongoing star formation (Tinsley 1971; Madau et al. 1996, 1998), the optical and NIR luminosities provide constraints on more evolved stellar populations (Hunter et al. 1982). This can be used, in principle, to derive the evolution of such basic galaxy properties as the stellar mass 
function (see e.g. Drory et al. 2005, and references therein), the star-formation rate density (see e.g. Pérez-González et al. 2005, and references therein), or the specific star-formation rate (see e.g. Feulner et al. 2005, and references therein). The determination of these quantities, however, is based on assumptions, e.g., on the shape of the initial mass function or on the details in modeling the stellar population like age, chemical composition, and star formation history. Hence, studying the LF at different wavelengths and cosmic epochs offers a more direct approach to the problem of galaxy evolution.

As the LF is one of the fundamental observational tools, the amount of work dedicated by different groups to derive accurate LFs is substantial. Based on either spectroscopic redshifts, drop-out techniques, or photometric redshifts, it has been possible to derive luminosity functions at different redshifts in the ultraviolet \& blue bands (Baldry et al. 2005; Croton et al. 2005; Arnouts et al. 2005; Budavári et al. 2005; Treyer et al. 2005; see also Gabasch et al. 2004a, and references therein), in the red bands (Lin et al. 1996, 1997; Brown et al. 2001; Shapley et al. 2001; Wolf et al. 2003; Chen et al. 2003; Ilbert et al. 2005; Dahlen et al. 2005; Trentham et al. 2005), as well as in the near-IR bands (Loveday 2000; Kochanek et al. 2001; Cole et al. 2001; Balogh et al. 2001; Drory et al. 2003; Huang et al. 2003; Feulner et al. 2003; Pozzetti et al. 2003; Dahlen et al. 2005).

The evolution of the characteristic luminosity and density of galaxy populations can be analyzed by fitting a Schechter function (Schechter 1976) to the LF. The redshift evolution of the three free parameters of the Schechter function - the characteristic magnitude $M^{*}$, the density $\phi^{*}$, and the faint-end slope $\alpha$ - can be used to quantitatively describe the change of the LF as a function of redshift. Unfortunately, the Schechter parametrization of the LF cannot account for possible excesses at the bright and faint ends or at other subtle shape deviations. Furthermore, the Schechter parameters are highly correlated, making it challenging, but not impossible, to clearly separate the evolution of the different parameters (see e.g. Andreon 2004 , for a discussion).

The evolution of the LFs is also very suitable to constrain the free parameters of theoretical models (e.g. semi-analytical or smoothed particle hydrodynamics models). Ideally a comparison between model predictions and observations should be done simultaneously for different wavebands (UV, optical, NIR) and for different redshift slices, as different stellar populations are involved in generating the flux in the different bands. Therefore, the FDF (Heidt et al. 2003) provides a unique testing ground for model predictions, since the depth and the covered area allow relatively precise LF measurements from the UV to the $z^{\prime}$ bands up to high redshift in a very homogeneous way.

In this paper we extend the measurements of the blue luminosity functions presented in Gabasch et al. (2004a, hereafter FDFLF I) to the red $r^{\prime}, i^{\prime}$, and $z^{\prime}$ bands. In Sect. 2 we derive the LFs and show the best-fitting Schechter parameters $M^{*}, \phi^{*}$, and $\alpha$ in the redshift range $0.5<z<3.5$. We also present a detailed analysis of the slope of the LF as a function of redshift and wavelength. Furthermore, we analyze the contributions of different SED types to the overall LF and present the evolution of the type-dependent luminosity density up to redshift $z \sim 2$.
Section 3 shows a parametric analysis of the redshift evolution of the LF, whereas comparisons with the LFs of other surveys and then with model predictions are given in Sect. 4 and in Sect. 5, respectively. We summarize our work in Sect. 6.

Throughout this paper we use $\mathrm{AB}$ magnitudes and adopt a $\Lambda$ cosmology with $\Omega_{\mathrm{M}}=0.3, \Omega_{\Lambda}=0.7$, and $H_{0}=70 \mathrm{~km} \mathrm{~s}^{-1} \mathrm{Mpc}^{-1}$.

\section{Luminosity functions in the $r^{\prime}, i^{\prime}$, and $z^{\prime}$ bands}

The results presented in this paper are all based on the deep part of the $I$-band selected catalog of the FDF (Heidt et al. 2003), as introduced in FDFLF I. Galaxy distances were determined by the photometric redshift technique (Bender et al. 2001 ) with a typical accuracys of $\Delta z /\left(z_{\text {spec }}+1\right) \approx 0.03$, if compared to the spectroscopic sample (Noll et al. 2004; Böhm et al. 2004) of more than 350 objects. To derive the absolute magnitude for a given band (which will be briefly summarized below) we used the best fitting SED as determined by the photometric redshift code, thus reducing possible systematic errors that could be introduced by using $\mathrm{k}$-corrections applied to a single observed magnitude. To account for the fact that some fainter galaxies are not visible in the whole survey volume, we performed a $V / V_{\max }$ (Schmidt 1968) correction. The errors of the LFs were calculated by means of Monte-Carlo simulations and included the photometric redshift error of every single galaxy, as well as the statistical error (Poissonian error). To derive precise Schechter parameters, we limited our analysis of the LF to the magnitude bin where $V / V_{\max } \leq 3$. We also show the uncorrected LF in the various plots as open circles. We did not assume any evolution of the galaxies within the single redshift bins, since the number of galaxies and the distance determination based on photometric redshifts would not be able to constrain it. The redshift binning was chosen such that we have good statistics in the various redshift bins and so that the influence of redshift clustering was minimized. In order to have good statistics at the bright end (rare objects) of the LF, we had to slightly change some of the redshift bins if compared to FDFLF I. The new redshift binning, together with the number of galaxies in every bin, is shown in Table 1. As can be seen from Table 1, the redshift intervals are approximately the same size in $\ln (1+z)$, and most of the results we are going to discuss in this paper are based on 700-1000 galaxies per redshift bin.

\subsection{The slope of the $L F$ as a function of redshift}

To investigate the redshift evolution of the faint-end slope of the LF, we fitted a three-parameter Schechter function $\left(M^{*}, \phi^{*}\right.$, and $\alpha$ ) to the data points. The best fitting slope $\alpha$ and the corresponding $1 \sigma$ errors for the 3 wavebands are reported in Table 2 for the various redshift bins.

It can be inferred from Table 2 that there is only marginal evidence of a change of $\alpha$ with redshift, at least up to $z \sim 2$ where we are able to sample the LF to a suitable depth. Under the assumption that $\alpha$ does not depend on redshift, Table 3 (upper part) yields the slopes' best error-weighted values in the redshift range from $\langle z\rangle \sim 0.65$ to $\langle z\rangle \sim 1.6$ (also including the higher redshift bins changes $\alpha$ only marginally). Since the 
Table 1. Number of galaxies in the FDF for the redshift intervals used for computing the LFs. Note that we derived the LF in all redshift bins, but excluded the lowest $(z<0.45)$ and highest redshift bins $(z>3.81)$ from our analysis of the LF evolution, since the lowest redshift bin corresponds to too small a volume, while the $z>3.81$ bin suffers from extrapolation errors.

\begin{tabular}{ccr}
\hline \hline $\begin{array}{c}\text { Redshift } \\
\text { interval }\end{array}$ & $\begin{array}{c}\text { Number } \\
\text { of galaxies }\end{array}$ & $\begin{array}{r}\text { Fraction } \\
\text { of galaxies }\end{array}$ \\
\hline $0.00-0.45$ & 808 & $14.54 \%$ \\
$0.45-0.85$ & 1109 & $19.95 \%$ \\
$0.85-1.31$ & 1029 & $18.51 \%$ \\
$1.31-1.91$ & 880 & $15.83 \%$ \\
$1.91-2.61$ & 816 & $14.68 \%$ \\
$2.61-3.81$ & 718 & $12.92 \%$ \\
$>3.81$ & 196 & $3.53 \%$ \\
unknown & 2 & $0.04 \%$ \\
\hline
\end{tabular}

slopes in all bands are very similar, we derived a combined slope of $\alpha_{r^{\prime}} \& i^{\prime} \& z^{\prime}=-1.33 \pm 0.03$ (Table 3, lower part).

Almost all of the slopes listed in Table 2 are compatible within $1 \sigma-2 \sigma$ with $\alpha=-1.33 \pm 0.03$. Therefore, we fixed the slope to this value for the subsequent analysis. Please note that this slope is steeper than for the blue bands $\left(\alpha_{\mathrm{UV} \& u^{\prime}}=-1.07\right.$ and $\alpha_{g^{\prime} \& B}=-1.25$ ), but it follows the trend observed in FDFLF I: with increasing wavelength the slope steepens; i.e. the ratio of faint to bright galaxies increases. This trend is illustrated best in Fig. 1, where we combine the results derived in FDFLF I with those of this work and plot the wavelength dependence of the LF slope. As we will show in Sect. 2.3, this effect can be explained by the contribution of different galaxy populations to the overall LF in the various wavebands.

\subsection{The I selection versus $I+B$ selection}

We checked the dependence of our results on the selection band by comparing the $I$-band selected catalog and the $I+B$ selected FDF catalog. The combined catalog has been described in Heidt et al. (2003), and it reaches limiting magnitudes of $I \sim 26.8$ and $B \sim 27.6$. In the combined sample, $M^{*}$ agrees within its $1 \sigma$ errors with the values derived from the $I$-band catalog only. The slope $\alpha$ tends to be slightly steeper in the combined sample, but not by more than $1 \sigma$. The larger number of objects in the combined catalog mostly influences the characteristic density $\phi^{*}$, which is a factor of 1.05 to 1.20 larger (depending on the redshift bin). Given the errors of $\phi^{*}$, this is on the order of $1 \sigma$ to $2 \sigma$.

\subsection{The slope of the $L F$ as a function of wavelength}

To better understand the filter-dependence of the LF slope shown in Fig. 1, we analyzed the contribution of different galaxy types to the overall LF. Thus, we subdivided our galaxy sample into four SED types and analyzed the typedependent LF; i.e. we determined the contribution of a typical SED type to the overall LF. The SEDs were mainly grouped according to the UV- $K$ color (see Fig. 2): for increasing spectral type (SED type $1 \rightarrow$ SED type 4 ) the SEDs become bluer; i.e. the UV flux (and thus the recent star formation rate) increases if compared to the $K$-band flux. Pannella et al. (2005) analyze the morphology of about 1400 galaxies in the FDF down to $I \sim 25 \mathrm{mag}$ on HST (ACS) data, and find a good correlation between the four main SED types and the morphology of the galaxies, at least up to redshift $z \sim 1.5$.

The four SED types also show a sequence in the restframe $U-V$ color that is often used to discriminate between blue and red galaxies (see e.g. Giallongo et al. 2005, and references therein). As the restframe $U-V$ color includes the $4000 \AA$ break, it is quite sensitive to such galaxy properties as age and star formation. The $U-V$ color lies in the range between 2.3-1.9, 2.0-1.6, 1.6-0.9, and 0.9-0 for SED type 1, 2, 3, and 4, respectively. Therefore, in a rough classification one can refer to SED types 1 and 2 (SED type 3 and 4 ) as red (blue) galaxies. We used the same SED cuts at all redshifts (see below); i.e. we did not use the time evolution of the galaxy color bimodality (see e.g. Giallongo et al. 2005) to redefine the main SED type of a galaxy as a function of redshift.

We show the LFs for the four SED types in three redshift intervals in Fig. 3: $0.45<z \leq 0.85,0.85<z \leq 1.31$, and $1.31<z \leq 1.91$. The SED type increases from the left panel to the right panel; i.e. the extremely star-forming galaxies are shown in the rightmost panel. The LFs for the different filters are color-coded and denoted in the upper part of the various panels. We show every LF to the limiting magnitude where the $V / V_{\max }$ begins to contribute by at most a factor of 1.5 , which is more conservative as for the overall $\mathrm{LF}\left(V / V_{\max } \leq 3\right.$ for every bin). For clarity, a Schechter function fit to the data is included.

First of all, it is clear from Fig. 3 that the faint-end of the LF is always dominated by SED type 4 galaxies. This is true for all analyzed bands. If we focus on the bright end of the SED type 4 LFs, we only see a relatively small variation between the different filters. On the other hand, the difference between the filters for SED type 1 (for the bright end) is very large. Although (because of the low number density) SED type 1 does not contribute at all to the faint-end of the LFs, the picture changes for the bright end. While for the bright end of the LF in the $U V$ (black line), SED type 1 and 4 galaxies have about the same number density, in the red bands SED type 1 galaxies dominate the LF.

This trend applies for all three redshift bins, although it is more pronounced at lower redshift. It explains the change of the LF slope naturally as a function of waveband. This can be best seen in Fig. 4 where we concentrate on only two filters. There we show the Schechter functions fitted to the LFs in the UV, as well as in the $i^{\prime}$-band for the redshift intervals $0.45<z \leq 0.85,0.85<z \leq 1.31$, and $1.31<z \leq 1.91$. We plot the single Schechter functions for all four SED types, as well as for the overall LF. In the UV the overall LF is completely dominated by the SED type 4 galaxies. On the other hand, the overall LF in the $i^{\prime}$-band is mainly dominated by SED type 1 to type 3 at the bright end, and SED type 4 at the faint-end. This results in a steeper slope for the overall LF.

Please note that in Figs. 3 and 4 we show the SED type LFs and Schechter functions to the limiting magnitude where the $V / V_{\max }$ begins to contribute by at most a factor of 1.5 , which is more conservative than for the overall LF, for which we allow a 
Table 2. Slope of the LF for all wavelengths and all redshifts as derived from a 3-parameter Schechter fit.

\begin{tabular}{cccc}
\hline \hline$z$ & $\alpha\left(r^{\prime}\right)$ & $\alpha\left(i^{\prime}\right)$ & $\alpha\left(z^{\prime}\right)$ \\
\hline $0.45-0.85$ & $-1.37(+0.04-0.04)$ & $-1.37(+0.04-0.03)$ & $-1.39(+0.04-0.04)$ \\
$0.85-1.31$ & $-1.25(+0.06-0.04)$ & $-1.27(+0.06-0.05)$ & $-1.34(+0.06-0.04)$ \\
$1.31-1.91$ & $-1.30(+0.16-0.09)$ & $-1.50(+0.13-0.10)$ & $-1.45(+0.12-0.09)$ \\
$1.91-2.61$ & $-1.01(+0.15-0.14)$ & $-1.03(+0.17-0.14)$ & $-0.97(+0.17-0.12)$ \\
$2.61-3.81$ & $-0.98(+0.17-0.17)$ & $-1.03(+0.15-0.13)$ & $-1.01(+0.15-0.13)$ \\
\hline
\end{tabular}

Table 3. In the upper part of the table, we show the slope $\alpha$ of the luminosity functions for the different wavebands as determined from an error-weighted fit to the data, under the assumption that $\alpha(z)=$ const. In the lower part of the table, we show the best values of $\alpha$ after combining the data of all bands.

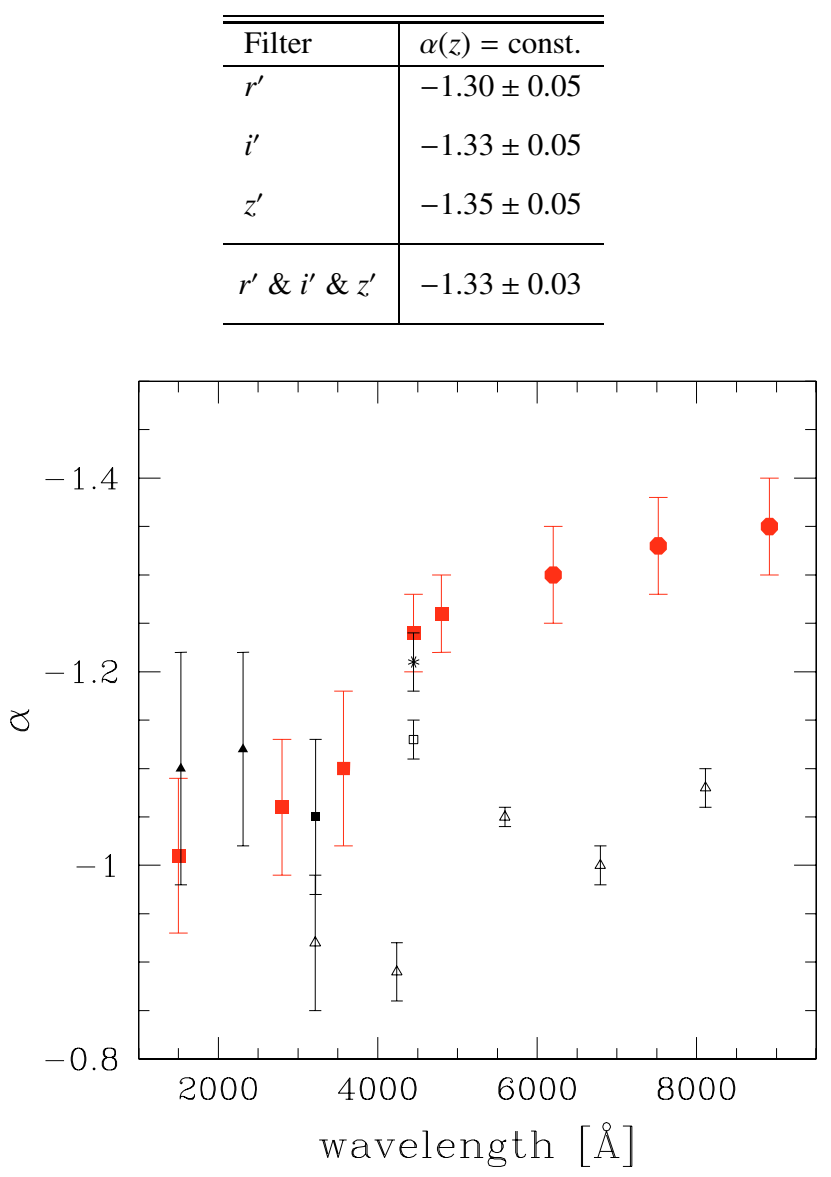

Fig. 1. Slope of the LF as a function of wavelength. The filled red squares denote the values derived in FDFLF I, whereas the filled red dots are taken from this work (Table 3, upper part). Local slope values (black) are shown as filled squares (Baldry et al. 2005), open squares (Driver et al. 2005), filled triangles (Budavári et al. 2005), open triangles (Blanton et al. 2003), and as an asterisk (Norberg et al. 2002).

correction factor of 3. Furthermore, all Schechter functions in Fig. 4 are fits to the data points. This is also true for the overall Schechter function, which is not the sum of the individual SED type Schechter functions, and this explains why, at the bright end, the overall Schechter function is below individual SED type Schechter functions in some plots.

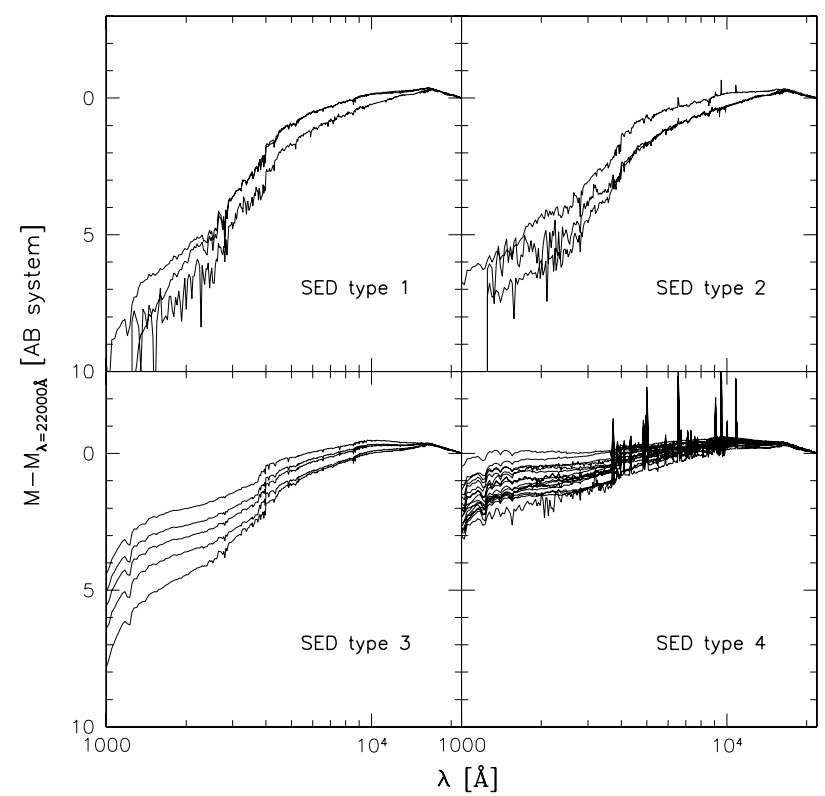

Fig. 2. SEDs grouped according to their spectral type. See text for details.

Another interesting aspect found from Fig. 4 is the fast decrease in number density of bright SED type 1 to 3 galaxies if compared to SED type 4 galaxies (for increasing redshift). Therefore at high redshift $(z \sim 2)$, SED type 4 galaxies also start to dominate the overall $i^{\prime}$-band LF. This can be seen best if one follows the redshift evolution of the type-dependent $l u$ minosity density (LD), i.e. the integrated light emitted by the different SED types. The results (for the UV and $i^{\prime}$ bands) are shown in Fig. 5. We calculated the LD as described in Gabasch et al. (2004b). First, we derived the LD at a given redshift by summing the completeness-corrected (using a $V / V_{\max }$ correction) luminosity of every single galaxy up to the absolute magnitude limits. Second, we applied a further correction (to zero galaxy luminosity) ZGL, to take the missing contribution to the LD of the fainter galaxies into account. To this end we used the best-fitting Schechter function for a slope $\alpha$ constant with redshift. For every SED type, we derived $\alpha(z)=$ const. by an error-weighted averaging of the slopes given in Table 5. This resulted in slopes between $\alpha=-0.98$ and $\alpha=-1.25$. For the FDF, the ZGL corrections are at most $22 \%$ in size (see last column in Table 4). The small ZGL correction employed here stems from the faint magnitude limits probed by our deep FDF data set and the relatively flat slopes of the Schechter function. Errors were computed from Monte Carlo simulations that take 


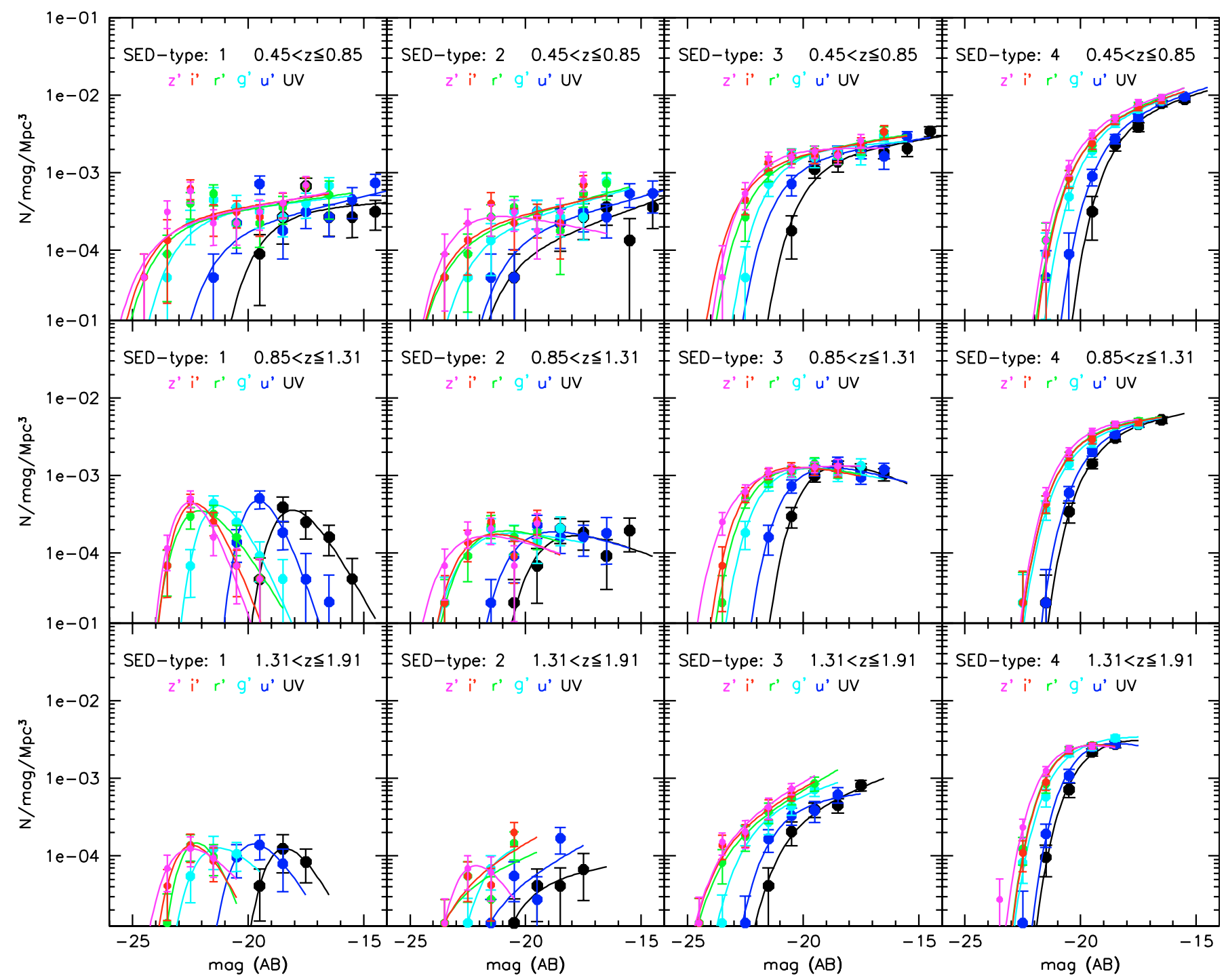

Fig. 3. LFs for the four SED types in the redshift intervals $0.45<z \leq 0.85$ (upper panels), $0.85<z \leq 1.31$ (middle panels), and $1.31<z \leq 1.91$ (lower panels): SED type increases from left to right. The filters are color-coded and denoted in the upper part of the various panels. For clarity, the three-parameter Schechter function fit to the data is shown as a solid line with the same color coding as the LF.

the probability distributions of the photometric redshifts and the Poissonian error into account.

As shown on the left panel of Fig. 5, the contribution of type 1 and 2 galaxies to the UV LD is negligible at all analyzed redshifts. The SED types 3 and 4 completely dominate the UV output, and although the number density of these galaxies decreases with increasing redshift, the luminosity density (and thus the SFR) increases.

If we analyze the $i^{\prime}$-band $\mathrm{LD}$, in the lowest redshift bin SED types 1 and 3 dominate (by a factor of about three if compared to types 2 and 4) and have about the same LD. At higher redshifts the relative contribution of the different SED types changes, because the LD of types 1 and 2 galaxies decreases with increasing redshift, and SED types 3 and 4 take over.

A detailed analysis of the type-dependent LF will be presented in a future paper (Gabasch et al., in preparation), where we combine the $I$-band selected MUNICS catalog (MUNICS IX, Feulner et al., in preparation, 900 $\operatorname{arcmin}^{2}$ ) with the FDF ( $\left.40 \operatorname{arcmin}^{2}\right)$ catalog. This overcomes the small volume of the FDF at lower redshift, making it possible to also include rare bright objects in the analysis of the LF. First results in the MUNICS fields will be presented in MUNICS IX.

\subsection{The redshift evolution of the LFs}

In this section we analyze the LF by means of a Schechter function fit with a fixed slope of $\alpha=-1.33$. In Figs. 6 and 7, we present the LFs in the $r^{\prime}$-band and in the $i^{\prime}$-band, while the results for the $z^{\prime}$-band can be found in Fig. 8. The filled (open) symbols denote the LF with (without) completeness correction. The solid lines show the Schechter function fitted to the luminosity function. The best fitting Schechter parameter, the redshift binning, as well as the reduced $\chi^{2}$, are also listed in each figure. The values of the reduced $\chi^{2}$ are very good for all redshift bins below $z \sim 2$. We do not fit our lowest redshift bin data $(\langle z\rangle \sim 0.3)$ with a Schechter function, because the volume 

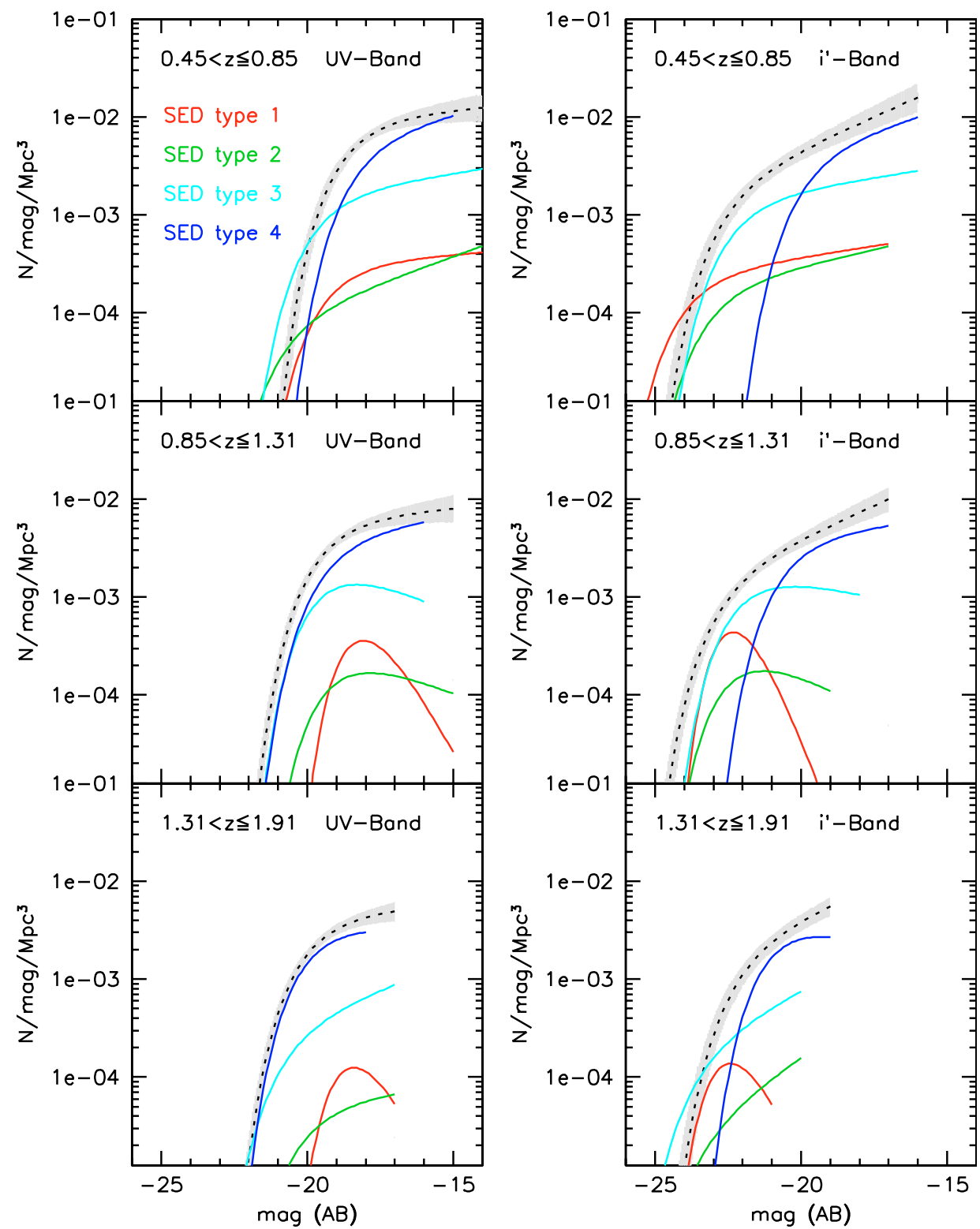

Fig. 4. Schechter functions fitted to the LFs in the UV (left panels), as well as in the $i^{\prime}$-band (right panels) for the redshift intervals $0.45<z \leq 0.85$ (upper panels), $0.85<z \leq 1.31$ (middle panels), and $1.31<z \leq 1.91$ (lower panels). The solid lines show the best-fitting Schechter functions for the four SED types. The SED type is color-coded and denoted in the upper left panel. The dotted black line shows the total LF (as fitted to the data), whereas the shaded region represents the corresponding $1 \sigma$ error of the latter. For the slope values of the different SED types, see Table 5. Please note that we show the SED type LFs to the limiting magnitude where the $V / V_{\max }$ begins to contribute by at most a factor of 1.5 , which is more conservative than for the overall LF, for which we allow a correction factor of 3.

is too small. For comparison we also show the local LF derived by Blanton et al. (2003) in the SDSS (see also Fig. 9). The best fitting Schechter parameters and corresponding $1 \sigma$ errors are summarized in Tables 6-8 for the $r^{\prime}, i^{\prime}$, and $z^{\prime}$ bands. Even without fitting Schechter functions to the data, it is obvious that the evolution in characteristic luminosity and number density between redshifts $\langle z\rangle=0.6$ and $\langle z\rangle=3.2$ is very moderate, if compared to the evolution in the blue bands.

\section{Parameterizing the evolution of the LFs}

To better quantify the redshift evolution of the LFs, we used the method introduced in FDFLF I. We parameterized the evolution of $M^{*}$ and $\phi^{*}$ with redshift assuming the following simple relations:

$$
\begin{aligned}
M^{*}(z) & =M_{0}^{*}+a \ln (1+z), \\
\phi^{*}(z) & =\phi_{0}^{*}(1+z)^{b}, \text { and } \\
\alpha(z) & =\alpha_{0} \equiv \text { const. }
\end{aligned}
$$

We then derived the best fitting values for the free parameters $a$, $b, M_{0}^{*}$, and $\phi_{0}^{*}$ by minimizing the $\chi^{2}$ of

$$
\begin{aligned}
\chi^{2} & =\chi^{2}\left(a, b, M_{0}^{*}, \phi_{0}^{*}\right) \\
& =\sum_{j=1}^{N_{j}} \sum_{i=1}^{N_{i}} \frac{\left[\phi\left(M_{i j}\right)-\Psi\left(M_{i j}, z_{j}, a, b, M_{0}^{*}, \phi_{0}^{*}\right)\right]^{2}}{\sigma_{i j}^{2}},
\end{aligned}
$$




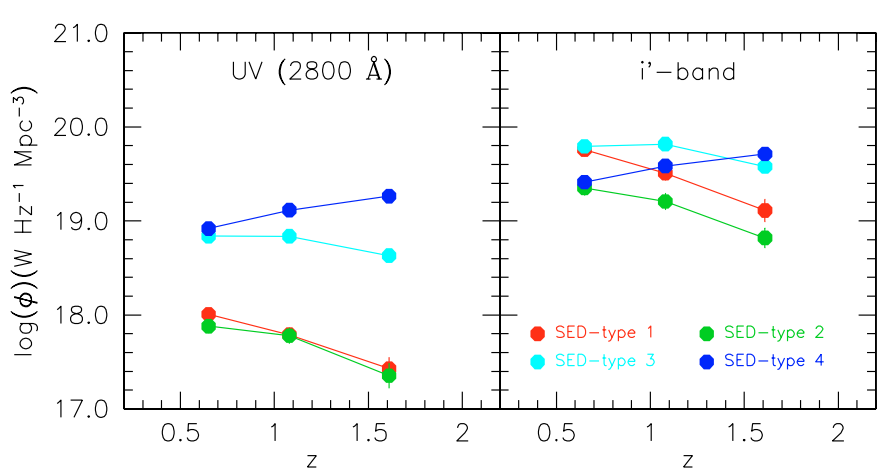

Fig. 5. Luminosity densities in the UV (left panel) and $i^{\prime}$ (right panel) bands for the four SED types in the redshift intervals $0.45<z \leq 0.85$, $0.85<z \leq 1.31$, and $1.31<z \leq 1.91$. The luminosity densities are completeness-corrected to zero luminosity (ZGL, see text for details). The values are listed in Table 4.

for the galaxy number densities in all magnitude and redshift bins simultaneously (for more details see FDFLF I). The free parameters of the evolutionary model are constrained for three different cases:

- Case 1: FDF LFs between redshift $\langle z\rangle \sim 0.65$ and $\langle z\rangle \sim 2.26$ are used.

- Case 2: FDF LFs between redshift $\langle z\rangle \sim 0.65$ and $\langle z\rangle \sim 3.21$ are used.

- Case 3: FDF LFs between redshift $\langle z\rangle \sim 0.65$ and $\langle z\rangle \sim 2.26$ as well as the local LF of Blanton et al. (2003) are used.

As can be seen from Fig. 1, the slopes of the red LFs derived by Blanton et al. (2003, SDSS Data release 1) are much shallower than those derived in this work and in a previous work of the same author (Blanton et al. 2001, SDSS EDR). Blanton et al. (2003) argue that the difference in the $r$-band LF (between Blanton et al. 2001; and Blanton et al. 2003) stems only from the inclusion of luminosity evolution within the covered redshift range. Very recently Driver et al. (2005) showed that the $B$-band LF derived from the Millennium Galaxy Catalogue (MGC, which is fully contained within the region of the SDSS) is inconsistent with the SDSS $z=0$ result of Blanton et al. (2003) by more than $3 \sigma$. On the other hand, the $M^{*}$ value of the $B$-band LF of Driver et al. (2005) is consistent with those derived by Blanton et al. (2001) after that $\phi^{*}$ has been renormalized according to Liske et al. (2003). Once corrected, the Blanton et al. (2001) B-band LF agrees well with the MGC, the 2dFGRS, and the ESO Slice Project estimates. Driver et al. (2005) conclude that the discrepancy between the MGC and the Blanton et al. (2003) LFs is complex, but caused predominantly by a color bias within the SDSS. They also conclude that the color selection bias might be a general trend across all filters.

In Fig. 10 we compare the local Schechter functions as given by Blanton et al. (2003) and Blanton et al. (2001, $\phi^{*}$ has been renormalized according to Liske et al. 2003) for the $r^{\prime}, i^{\prime}$, and $z^{\prime}$ bands. Although there is reasonably good agreement between the LFs if one focuses on the bright part $(M \lesssim$ $-20)$, they disagree at fainter magnitudes. On the other hand, the slope of the LF is strongly dependent on the depth of the survey. The flux limit in the $r$-band selected SDSS survey is about $m_{r}<17.79$. A very rough estimate of the absolute limiting magnitude at the mean redshift of the survey $(\langle z\rangle=0.1)$ is therefore $M_{r^{\prime}} \approx-20$. This means that the faint-end of the LF as shown in Fig. 10 depends on the applied completeness correction (see also the discussion in Andreon 2004). Therefore, we decided to use only the bright part $(M \lessgtr-20)$ of the SDSS LFs to constrain the free evolutionary parameter of Case 3.

As the Schechter parameters are coupled, and $M^{*}$ and $\phi^{*}$ of Blanton et al. (2003) are derived for a different slope $\alpha$, we decided not to use $M^{*}$ and $\phi^{*}$ itself, but to reconstruct a magnitude binned luminosity function out of the Schechter values $M^{*}, \phi^{*}$, and $\alpha$ given in Blanton et al. (2003). Following the method described in Sect. 4 to visualize the errors of the literature LFs (shaded regions in the plots) we derived the 1-magbinned LF as shown in Fig. 10 (solid points). Figure 10 shows that a Schechter function fit to the SDSS data with a slope of $\alpha=-1.33$ (as derived from the FDF data) results in a reduced $\chi_{\text {red }}^{2} \sim 10$. This large $\chi_{\text {red }}^{2}$ increases the errorbars of the evolutionary parameter since we normalize the result of Eq. (2) to $\chi_{\text {red }}^{2} \sim 1$ before calculating the errors.

The $1 \sigma$ and $2 \sigma$ confidence levels of the evolution parameters $a$ and $b$ for the different filters and cases are shown in Fig. 11. These contours were derived by projecting the fourdimensional $\chi^{2}$ distribution to the $a-b$ plane, i.e. for given $a$ and $b$ we used the value of $M_{0}^{*}$ and $\phi_{0}^{*}$, which minimizes the $\chi^{2}(a, b)$.

For Case 1 (left panel) the errorbars of $a$ and $b$ are rather large; and although the best fitting values suggest a redshift evolution they are also compatible (within $2 \sigma$ ) with no evolution of $M^{*}$ and $\phi^{*}$. The error ellipses for Case 2 (middle panel) are smaller than in Case 1, and for the $r^{\prime}$-band LF, we see a luminosity and a density evolution on a $2 \sigma$ level. For the $i^{\prime}$-band and $z^{\prime}$-band LFs, we see only density evolution on a $2 \sigma$ level. Also including the local LF of Blanton et al. (2003) in the evolution analysis, as in Case 3 (left panel), we were able to derive $a$ and $b$ with higher precision since $M_{0}^{*}$ and $\phi_{0}^{*}$ are more restricted. The luminosity and density evolution is clearly visible on more than the $2 \sigma$ level. Please note that combining different datasets, such as the FDF and the SDSS, can introduce systematic errors due to the different selection techniques and calibration differences not being fully taken into account (see also discussion above). Nevertheless, a comparison of the FDF LFs with the SDSS Schechter functions in Fig. 9 shows relatively good agreement (at the bright end). Furthermore, a detailed comparison of the UV LFs of the FDF with the LF derived in large surveys e.g. Wolf et al. (2003, based on COMBO-17), Steidel et al. (1999, based on LBG analysis), Iwata et al. (2003); Ouchi et al. (2004, based on Subaru Deep Field/Survey) or pencil beam surveys e.g. Poli et al. (2001, based on both HDFs) presented in FDFLF I shows good agreement in the overlapping magnitude range at all redshifts. We are thus confident that the remaining systematic differences (e.g. due to the influence of large scale structure; LSS) must be small.

The values for the free parameters $a, b, M_{0}^{*}$, and $\phi_{0}^{*}$, as well as the associated errors, can be found in Table 9 . The evolution 
Table 4. Luminosity densities in the UV and $i^{\prime}$ bands for the four SED types. See also Fig. 5.

\begin{tabular}{|c|c|c|c|c|c|}
\hline Filter & SED type & Redshift & $\begin{array}{c}\text { Luminosity density } \\
\mathrm{W} \mathrm{Hz}^{-1} \mathrm{Mpc}^{-3}\end{array}$ & $\begin{array}{c}\text { Error } \\
\mathrm{W} \mathrm{Hz}^{-1} \mathrm{Mpc}^{-3} \\
\end{array}$ & $\begin{array}{c}\text { Completeness correction (ZGL) } \\
\% \\
\end{array}$ \\
\hline \multirow[t]{12}{*}{ UV (2800 ̊) } & \multirow[t]{3}{*}{1} & $0.45-0.85$ & $1.01 \mathrm{e}+18$ & $1.5 \mathrm{e}+17$ & 0.1 \\
\hline & & $0.85-1.31$ & $6.16 e+17$ & $1.0 \mathrm{e}+17$ & 0.7 \\
\hline & & $1.31-1.91$ & $2.68 \mathrm{e}+17$ & $7.6 e+16$ & 8.9 \\
\hline & \multirow[t]{3}{*}{2} & $0.45-0.85$ & $7.58 \mathrm{e}+17$ & $1.3 e+17$ & 0.5 \\
\hline & & $0.85-1.31$ & $6.00 \mathrm{e}+17$ & $1.2 \mathrm{e}+17$ & 2.0 \\
\hline & & $1.31-1.91$ & $2.26 \mathrm{e}+17$ & $7.0 \mathrm{e}+16$ & 9.0 \\
\hline & \multirow[t]{3}{*}{3} & $0.45-0.85$ & $6.88 \mathrm{e}+18$ & $7.9 e+17$ & 0.6 \\
\hline & & $0.85-1.31$ & $6.86 \mathrm{e}+18$ & $5.4 \mathrm{e}+17$ & 2.0 \\
\hline & & $1.31-1.91$ & $4.27 \mathrm{e}+18$ & $5.6 e+17$ & 4.7 \\
\hline & \multirow[t]{3}{*}{4} & $0.45-0.85$ & $8.29 \mathrm{e}+18$ & $5.1 e+17$ & 8.4 \\
\hline & & $0.85-1.31$ & $1.30 \mathrm{e}+19$ & $8.4 \mathrm{e}+17$ & 6.9 \\
\hline & & $1.31-1.91$ & $1.83 \mathrm{e}+19$ & $1.9 \mathrm{e}+18$ & 21.4 \\
\hline \multirow[t]{12}{*}{$i^{\prime}$} & \multirow[t]{3}{*}{1} & $0.45-0.85$ & $5.72 \mathrm{e}+19$ & $8.7 e+18$ & 0.3 \\
\hline & & $0.85-1.31$ & $3.22 \mathrm{e}+19$ & $5.4 \mathrm{e}+18$ & 1.5 \\
\hline & & $1.31-1.91$ & $1.29 \mathrm{e}+19$ & $3.5 e+18$ & 10.2 \\
\hline & \multirow[t]{3}{*}{2} & $0.45-0.85$ & $2.24 \mathrm{e}+19$ & $3.9 \mathrm{e}+18$ & 0.8 \\
\hline & & $0.85-1.31$ & $1.61 \mathrm{e}+19$ & $3.2 \mathrm{e}+18$ & 3.6 \\
\hline & & $1.31-1.91$ & $6.60 \mathrm{e}+18$ & $1.6 e+18$ & 11.6 \\
\hline & \multirow[t]{3}{*}{3} & $0.45-0.85$ & $6.21 \mathrm{e}+19$ & $6.9 e+18$ & 0.4 \\
\hline & & $0.85-1.31$ & $6.56 \mathrm{e}+19$ & $5.1 e+18$ & 1.6 \\
\hline & & $1.31-1.91$ & $3.79 \mathrm{e}+19$ & $5.4 \mathrm{e}+18$ & 7.4 \\
\hline & \multirow[t]{3}{*}{4} & $0.45-0.85$ & $2.59 \mathrm{e}+19$ & $1.5 \mathrm{e}+18$ & 5.8 \\
\hline & & $0.85-1.31$ & $3.84 \mathrm{e}+19$ & $2.4 \mathrm{e}+18$ & 5.7 \\
\hline & & $1.31-1.91$ & $5.17 \mathrm{e}+19$ & $4.5 e+18$ & 18.9 \\
\hline
\end{tabular}

Table 5. Slope of the UV (2800 ̊̊) and $i^{\prime}$ band LF for the different SED types from a 3-parameter Schechter fit. The Schechter functions are shown in Fig. 4.

\begin{tabular}{cccccc}
\hline \hline$z$ & Filter & $\alpha$ for SED type 1 & $\alpha$ for SED type 2 & $\alpha$ for SED type 3 & $\alpha$ for SED type 4 \\
\hline $0.45-0.85$ & UV & $-1.06(+0.16-0.10)$ & $-1.27(+0.08-0.04)$ & $-1.12(+0.11-0.07)$ & $-1.19(+0.13-0.11)$ \\
$0.45-0.85$ & $i^{\prime}$ & $-1.11(+0.15-0.02)$ & $-1.17(+0.22-0.03)$ & $-1.12(+0.12-0.11)$ & $-1.23(+0.07-0.10)$ \\
& & & & & \\
$0.85-1.31$ & UV & $+0.38(+0.60-0.37)$ & $-0.71(+0.62-0.27)$ & $-0.68(+0.17-0.15)$ & $-1.14(+0.12-0.08)$ \\
$0.85-1.31$ & $i^{\prime}$ & $+1.04(+0.65-0.68)$ & $-0.62(+0.79-0.32)$ & $-0.84(+0.15-0.13)$ & $-1.09(+0.11-0.06)$ \\
\hline
\end{tabular}

parameters $a, b, M_{0}^{*}$, and $\phi_{0}^{*}$ derived in Case 1, Case 2, and Case 3 all agree within $2 \sigma$. Most of the values differ only by $1 \sigma$ or less.

In Fig. 12 we illustrate the relative redshift evolution of $M^{*}$ for the different filters and different cases, while the relative redshift evolution of $\phi^{*}$ is shown in Fig. 13. Note that $a, b$, $M_{0}^{*}$, and $\phi_{0}^{*}$ were derived by minimizing Eq. (2) and not the differences between the (best fitting) lines and the data points in Figs. 12 and 13. As for the blue bands (FDFLF I), the simple parametrization of Eq. (1) is also able to describe the evolution of the galaxy LFs in the red bands very well.
Recently, Blanton et al. (2005) used the data of the SDSS Data Release 2 to analyze the very local LF $(0.00<z<0.05$; corrected for surface-brightness incompleteness) down to extremely low luminosity galaxies. They found that a Schechter function is an insufficient parametrization of the $\mathrm{LF}$, as there is an upturn in the slope of the LF for $M_{r}-5 \log \left(h_{100}\right)>-18$. In Fig. 9 we therefore compare the red FDF LFs in two redshift ranges $(\langle z\rangle=0.3$ and $\langle z\rangle=0.65)$ with the local Schechter functions as derived in the SDSS by Blanton et al. (2003) and Blanton et al. (2005). Considering the small volume covered by the FDF in the redshift bin $\langle z\rangle=0.3$ and the fact that we 

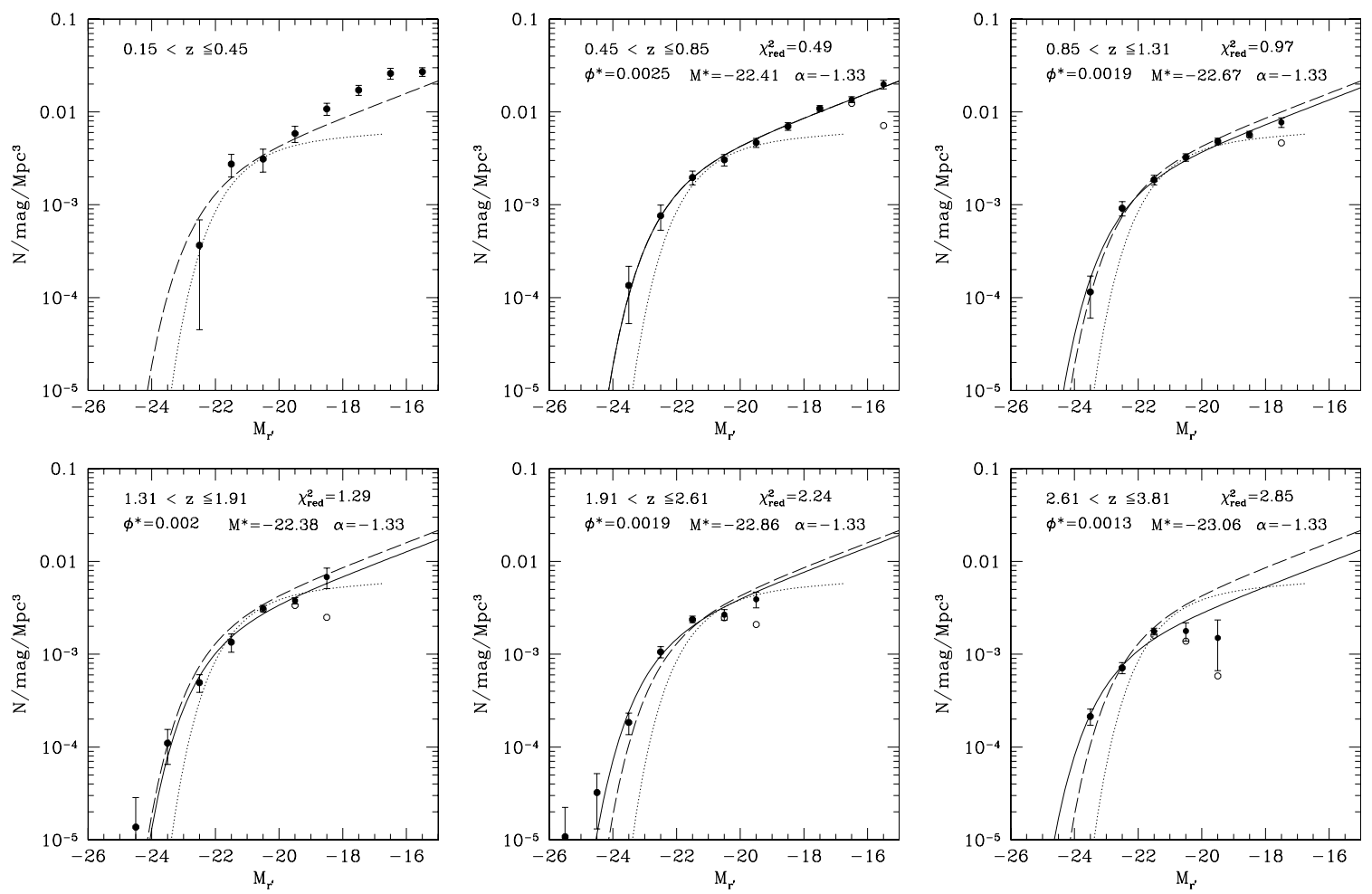

Fig. 6. The LFs in the $r^{\prime}$-band from low redshift $(\langle z\rangle=0.3$, upper left panel) to high redshift $(\langle z\rangle=3.2$, lower right panel). The filled (open) symbols show the LF corrected (uncorrected) for $V / V_{\max }$. The fitted Schechter functions for a fixed slope $\alpha$ are shown as solid lines. Note that we only fit the LFs from $\langle z\rangle=0.6$ to $\langle z\rangle=3.2$. The parameters of the Schechter functions can be found in Table 6 . The dotted line represents the local $r^{\prime}$-band LF derived from the SDSS (Blanton et al. 2003). The Schechter fit for redshift $\langle z\rangle=0.6$ is indicated as a dashed line in all panels.
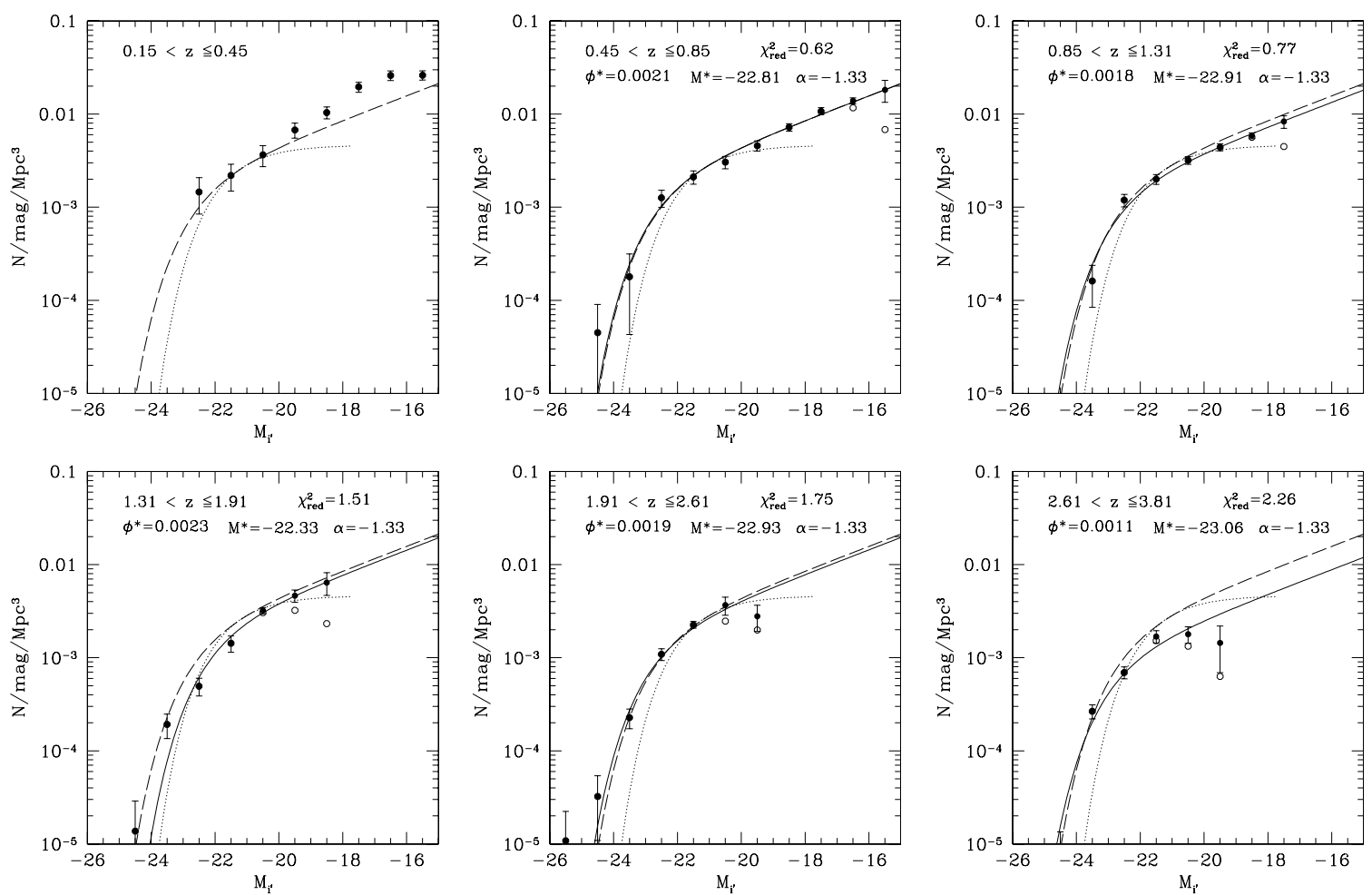

Fig. 7. The LFs in the $i^{\prime}$-band from low redshift $(\langle z\rangle=0.3$, upper left panel) to high redshift $(\langle z\rangle=3.2$, lower right panel). The filled (open) symbols show the LF corrected (uncorrected) for $V / V_{\max }$. The fitted Schechter functions for a fixed slope $\alpha$ are shown as solid lines. Note that we only fit the LFs from $\langle z\rangle=0.6$ to $\langle z\rangle=3.2$. The parameters of the Schechter functions can be found in Table 7. The dotted line represents the local $i^{\prime}$-band LF derived from the SDSS (Blanton et al. 2003). The Schechter fit for redshift $\langle z\rangle=0.6$ is indicated as a dashed line in all panels. 
Table 6. Schechter function fit in the $r^{\prime}$-band.

\begin{tabular}{cccc}
\hline \hline Redshift interval & $M^{*}(\mathrm{mag})$ & $\phi^{*}\left(\mathrm{Mpc}^{-3}\right)$ & $\alpha$ (fixed) \\
\hline $0.45-0.85$ & $-22.41+0.23-0.18$ & $0.0025+0.0002-0.0002$ & -1.33 \\
$0.85-1.31$ & $-22.67+0.14-0.13$ & $0.0019+0.0001-0.0001$ & -1.33 \\
$1.31-1.91$ & $-22.38+0.16-0.16$ & $0.0020+0.0002-0.0002$ & -1.33 \\
$1.91-2.61$ & $-22.86+0.13-0.11$ & $0.0019+0.0002-0.0002$ & -1.33 \\
$2.61-3.81$ & $-23.06+0.15-0.15$ & $0.0013+0.0002-0.0001$ & -1.33 \\
\hline
\end{tabular}

Table 7. Schechter function fit in the $i^{\prime}$-band.

\begin{tabular}{cccc}
\hline \hline Redshift interval & $M^{*}(\mathrm{mag})$ & $\phi^{*}\left(\mathrm{Mpc}^{-3}\right)$ & $\alpha$ (fixed) \\
\hline $0.45-0.85$ & $-22.81+0.23-0.24$ & $0.0021+0.0002-0.0002$ & -1.33 \\
$0.85-1.31$ & $-22.91+0.16-0.15$ & $0.0018+0.0001-0.0001$ & -1.33 \\
$1.31-1.91$ & $-22.33+0.21-0.18$ & $0.0023+0.0003-0.0003$ & -1.33 \\
$1.91-2.61$ & $-22.93+0.14-0.13$ & $0.0019+0.0002-0.0002$ & -1.33 \\
$2.61-3.81$ & $-23.06+0.10-0.09$ & $0.0011+0.0001-0.0001$ & -1.33 \\
\hline
\end{tabular}

Table 8. Schechter function fit in the $z^{\prime}$-band.

\begin{tabular}{cccc}
\hline \hline redshift interval & $M^{*}(\mathrm{mag})$ & $\phi^{*}\left(\mathrm{Mpc}^{-3}\right)$ & $\alpha$ (fixed) \\
\hline $0.45-0.85$ & $-23.06+0.25-0.21$ & $0.0022+0.0002-0.0002$ & -1.33 \\
$0.85-1.31$ & $-23.30+0.20-0.21$ & $0.0017+0.0002-0.0001$ & -1.33 \\
$1.31-1.91$ & $-22.71+0.18-0.17$ & $0.0020+0.0003-0.0002$ & -1.33 \\
$1.91-2.61$ & $-23.19+0.13-0.13$ & $0.0018+0.0002-0.0002$ & -1.33 \\
$2.61-3.81$ & $-23.42+0.10-0.13$ & $0.0010+0.0001-0.0001$ & -1.33 \\
\hline
\end{tabular}

see clustered spectroscopic redshifts at $z=0.22, z=0.33$, and $z=0.39$, the agreement between the LFs and the Schechter functions is relatively good for $M<-19$. For the fainter part, the measured number density disagrees with Blanton et al. (2003) and Blanton et al. (2005) in all three analyzed bands. If we do the same comparison at $\langle z\rangle=0.65$ where the FDF covers a relatively large volume minimizing the influence of LSS, the measured LFs also follow the very local Schechter function of Blanton et al. (2005) in the faint magnitude regime. Moreover, the upturn of the faint-end of the LF as found by Blanton et al. (2005) in the SDSS or by Popesso et al. (2005) in the RASSSDSS Galaxy Cluster Survey (see also Pérez-González et al. 2005 ), is also visible in the FDF data (at least at $\langle z\rangle=0.65$ ).

This upturn seems to be less pronounced in the UV (FDFLF I). A possible reason for this could again be the different contribution of the SED-type LFs presented in Fig. 4. In the red bands, the difference between the characteristic luminosities between the LFs for types 1, 2, 3 and 4, together with the dominance of the type-4 LF at the faint end, results in a dip at $M \sim-20$.

Although a Schechter function is an insufficient parametrization of the LF derived by Blanton et al. (2005), we used their results as local reference point to calculate the evolution of the LF in the various bands by minimizing Eq. (2). Due to the upturn of the faint-end of the local LF and the fact that our evolutionary model assumes a normal Schechter function, the reduced $\chi^{2}$ of Eq. (2) is on the order of 9. As we do not want to increase the number of free parameters by using a double Schechter function (at higher redshifts the data are not able to constrain a possible upturn in the LF), we increase the errors of $a, b, M_{0}^{*}$, and $\phi_{0}^{*}$. We do this by an appropriate scaling of the errors $\sigma_{i j}$ of Eq. (2) to obtain a reduced $\chi^{2}$ of unity. A comparison of the evolution parameters $a$ and $b$ with those derived in Case 3 shows that the evolution in the characteristic luminosity agrees with Case 3, but the evolution of the characteristic density decreases from $b \sim-0.7$ to $b \sim-0.5$ and therefore is closer to Case 1 and Case 2. However, a no-evolution hypothesis can be excluded on the $2 \sigma$ level in all three bands if the results of Blanton et al. (2005) are used as local reference points.

If we compare the evolutionary parameters $a$ and $b$ of the red bands with those derived in the blue bands (FDFLF I), the following trend can be seen: with increasing waveband the redshift evolution of $M^{\star}$ and $\phi^{\star}$ decreases. Furthermore, if we also include in our analysis the results obtained in the SDSS (Blanton et al. 2003), the brightening of $M^{\star}$ and the decrease in $\phi^{\star}$ for increasing redshift is still visible in the red bands at more than $3 \sigma$.

\section{Comparison with observational results from the literature}

To put the FDF results on the evolution of the LFs into perspective, we compared them to other surveys using the following approach. First we convert results from the literature to our cosmology $\left(\Omega_{\mathrm{M}}=0.3, \Omega_{\Lambda}=0.7\right.$, and $\left.H_{0}=70 \mathrm{~km} \mathrm{~s}^{-1} \mathrm{Mpc}^{-1}\right)$. 

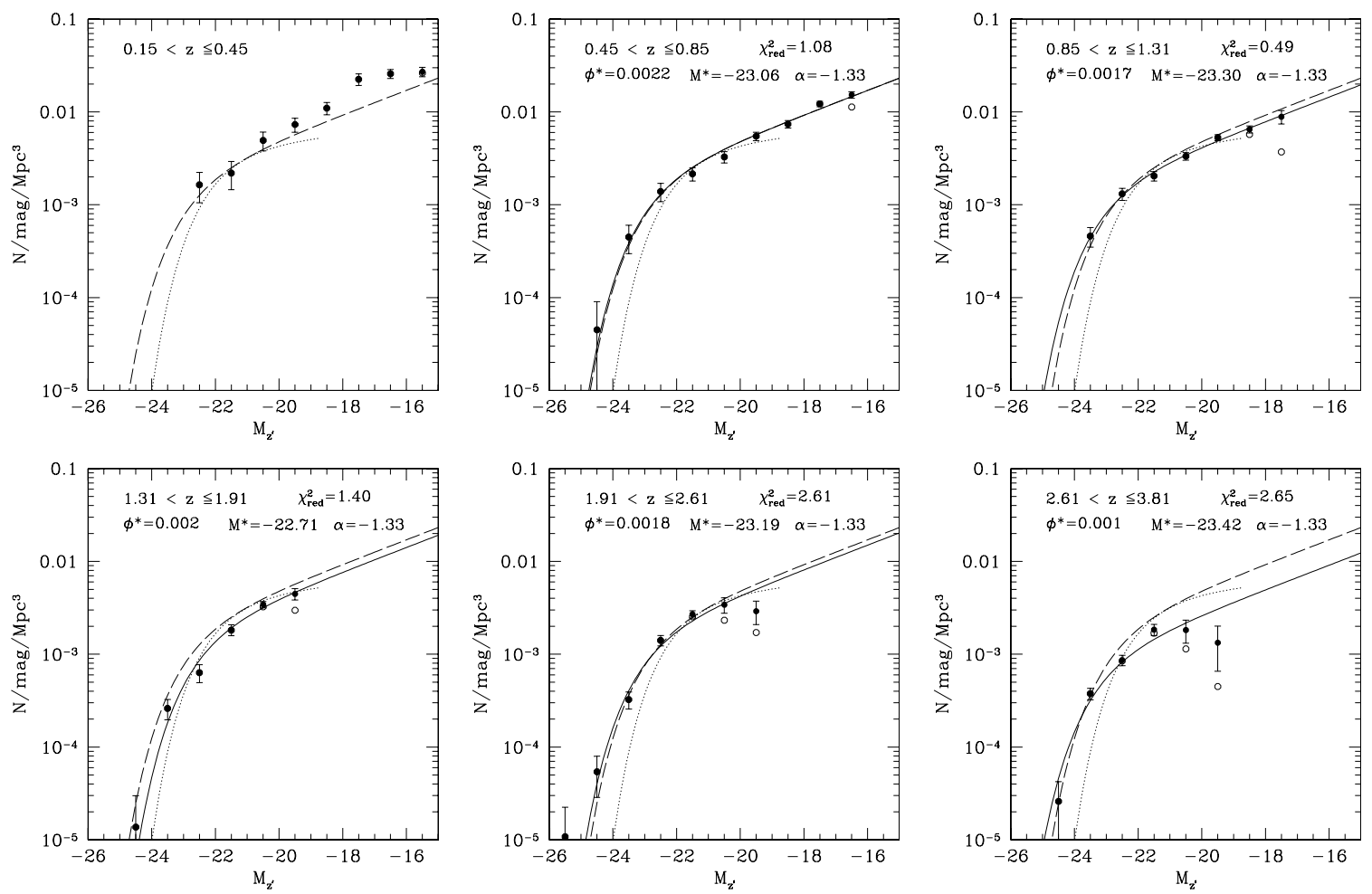

Fig. 8. The LFs in the $z^{\prime}$-band from low redshift $(\langle z\rangle=0.3$, upper left panel) to high redshift $(\langle z\rangle=3.2$, lower right panel). The filled (open) symbols show the LF corrected (uncorrected) for $V / V_{\max }$. The fitted Schechter functions for a fixed slope $\alpha$ are shown as solid lines. Note that we only fit the LFs from $\langle z\rangle=0.6$ to $\langle z\rangle=3$.2. The parameters of the Schechter functions can be found in Table 8. The dotted line represents the local $z^{\prime}$-band LF derived from the SDSS (Blanton et al. 2003). The Schechter fit for redshift $\langle z\rangle=0.6$ is indicated as a dashed line in all panels.
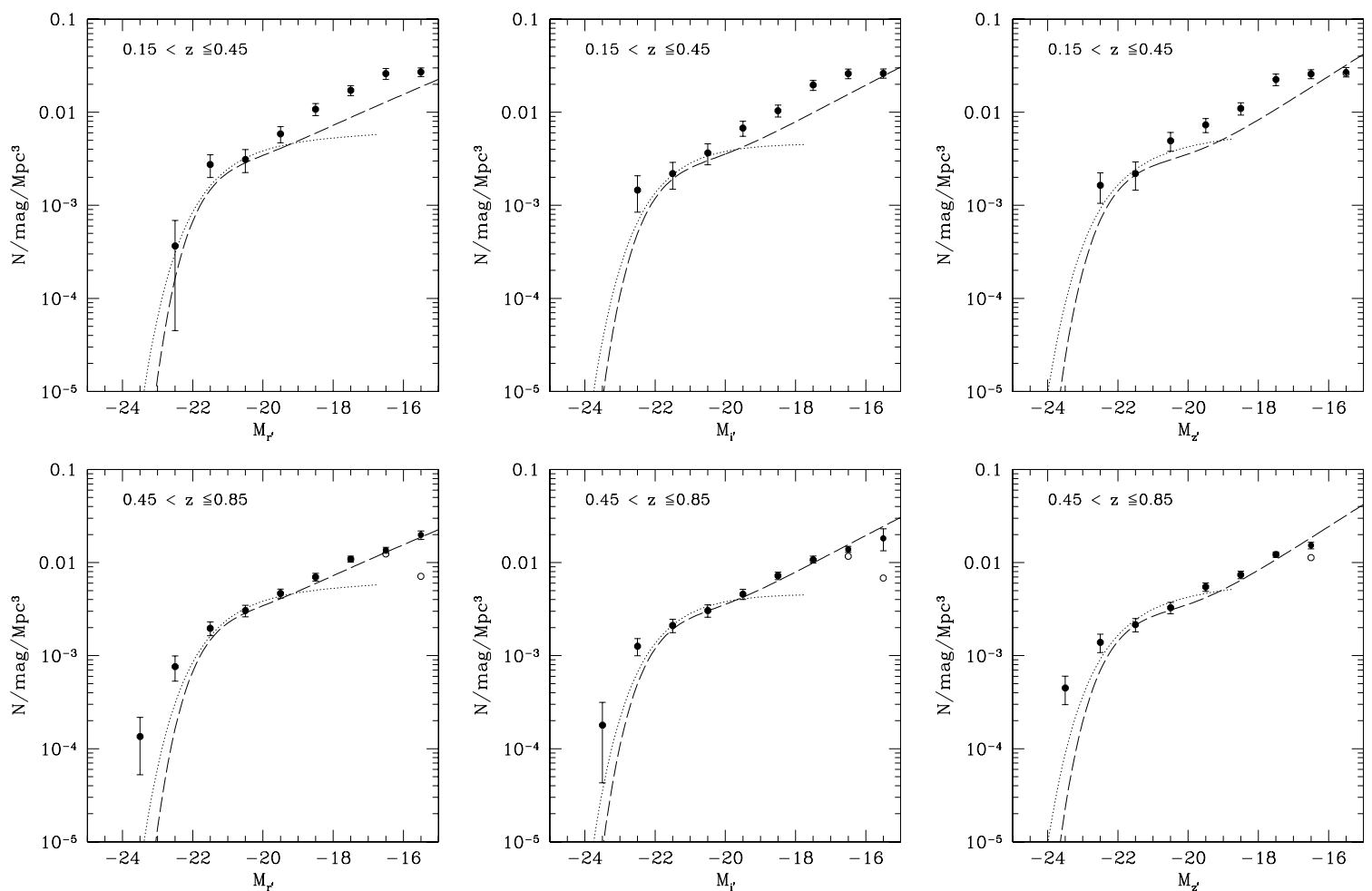

Fig. 9. Comparison of the red FDF LFs in the redshift range $\langle z\rangle=0.3$ (upper panels) and $\langle z\rangle=0.65$ (lower panels) with the local Schechter functions as derived in the SDSS by Blanton et al. (2003, dotted line) and Blanton et al. (2005, dashed line). The filled (open) symbols show the FDF LF corrected (uncorrected) for $V / V_{\max }$. 

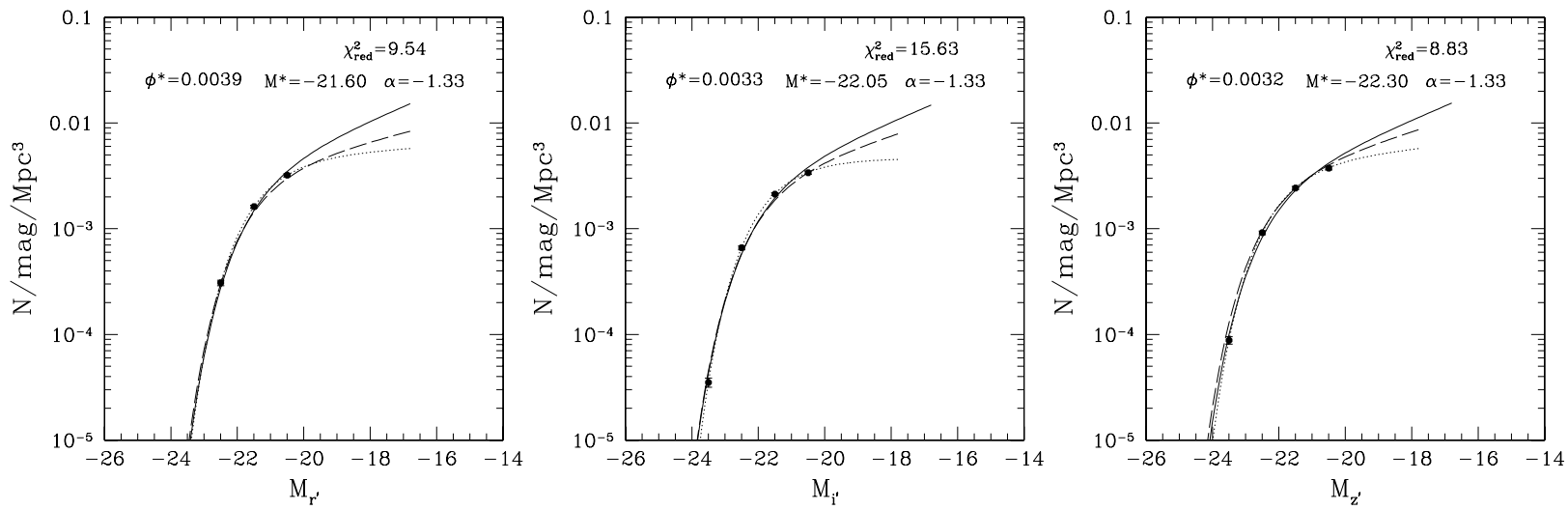

Fig. 10. The local LFs as given by Blanton et al. (2003) for the $r^{\prime}$ (left panel), $i^{\prime}$ (middle panel), and $z^{\prime}$ (left panel) bands. The dotted lines in all plots represent the best fitting Schechter function of Blanton et al. (2003). The solid points and the associated errorbars are derived by the Schechter values and corresponding errors of the latter (see text). The dashed lines represent the result of Blanton et al. (2001) after renormalizing $\phi^{*}$ according to Liske et al. (2003). We also fit a Schechter function (solid line) with a fixed slope of -1.33 as derived from the FDF data do the LF (solid dots). The corresponding $M^{*}, \phi^{*}$, and the reduced $\chi^{2}$ of the fit are also given in the figures.
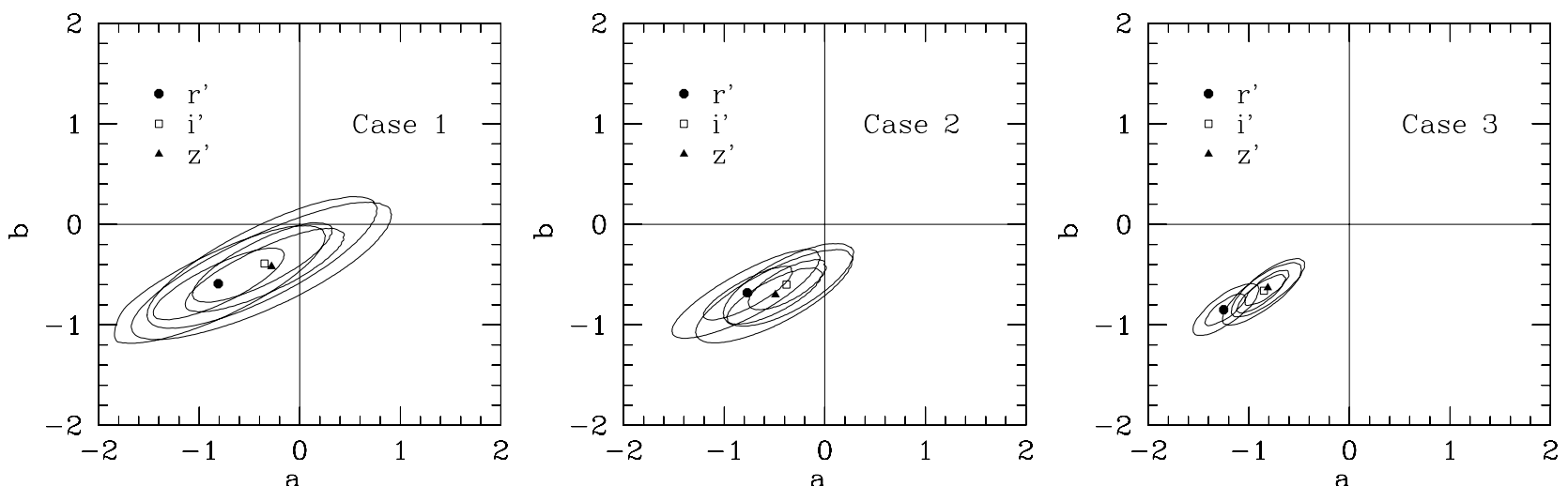

Fig. 11. $1 \sigma$ and $2 \sigma$ confidence levels of the parameters $a$ and $b$ in different bands $\left(r^{\prime}, i^{\prime}\right.$, and $\left.z^{\prime}\right)$ for the evolutionary model described in the text. Left panel: FDF LFs between redshift $\langle z\rangle \sim 0.65$ and $\langle z\rangle \sim 2.26$ are used (Case 1). The values for $a$ and $b$ can be found in Table 9. Middle panel: FDF LFs between redshift $\langle z\rangle \sim 0.65$ and $\langle z\rangle \sim 3.21$ are used (Case 2). The values for $a$ and $b$ can be found in Table 9. Right panel: FDF LFs between redshift $\langle z\rangle \sim 0.65$ and $\langle z\rangle \sim 2.26$ as well as the local LF of Blanton et al. (2003) are used (Case 3). The values for $a$ and $b$ can be found in Table 9.

Although this conversion may not be perfect (we can only transform number densities and magnitudes but lack knowledge of the individual magnitudes and redshifts of the galaxies), the errors introduced in this way are not large, so the method is suitable for our purpose. Second, in order to avoid uncertainties due to conversion between different filter bands, we always convert our data to the same band as the survey we want to compare with. Third, we try to use the same redshift binning as in the literature.

To visualize the errors of the literature LFs, we performed Monte-Carlo simulations using the $\Delta M^{*}, \Delta \phi^{*}$, and $\Delta \alpha$ given in the papers. In cases where all of these values could not be found in the paper, this is mentioned in the figure caption. We do not take into account any correlation between the Schechter parameters and assume a Gaussian distribution of the errors $\Delta M^{*}$, $\Delta \phi^{*}$, and $\Delta \alpha$. From 1000 simulated Schechter functions, we derived the region where $68.8 \%$ of the realizations lie. The resulting region, roughly corresponding to $1 \sigma$ errors, is shaded in the figures. The LFs derived in the FDF are also shown as filled and open circles. The filled circles are completeness corrected, whereas the open circles are not corrected. The redshift binning used to derive the LF in the FDF, as well as the literature redshift binning, is given in the upper part of every figure. Moreover, the limiting magnitude of the respective survey is indicated by the low-luminosity cut-off of the shaded region in all figures. If the limiting magnitude was not explicitly given, it was estimated from the figures in the literature.

A comparison of our FDF results with LFs based on spectroscopic distance determinations (Blanton et al. 2003, 2005; Lin et al. 1996, 1997; Brown et al. 2001; Shapley et al. 2001; Ilbert et al. 2005), as well as with LFs based on photometric redshifts (Wolf et al. 2003; Chen et al. 2003; Dahlen et al. 2005), follows:

\section{Blanton et al. (2003, 2005)}

In Fig. 9 we compare the red FDF LFs in two redshift regimes $(\langle z\rangle=0.3$ and $\langle z\rangle=0.65)$ with the local Schechter functions as derived in the SDSS by Blanton et al. (2003), and Blanton et al. (2005). As previously discussed, the agreement between the LFs and the Schechter functions is relatively good for 
Table 9. Evolution parameters according to Eq. (1).

\begin{tabular}{cccccc}
\hline \hline Filter & Case & $a$ & $b$ & $\begin{array}{c}M_{0}^{*} \\
(\mathrm{mag})\end{array}$ & $\begin{array}{c}\phi_{0}^{*} \\
\left(\mathrm{Mpc}^{-3}\right)\end{array}$ \\
\hline$r^{\prime}$ & Case 1 & $-0.81_{-0.41}^{+0.43}$ & $-0.59_{-0.23}^{+0.23}$ & $-21.89_{-0.42}^{+0.39}$ & $0.0033_{-0.0005}^{+0.0007}$ \\
$r^{\prime}$ & Case 2 & $-0.77_{-0.28}^{+0.30}$ & $-0.68_{-0.17}^{+0.17}$ & $-21.92_{-0.30}^{+0.30}$ & $0.0037_{-0.0005}^{+0.0005}$ \\
$r^{\prime}$ & Case 3 & $-1.25_{-0.10}^{+0.14}$ & $-0.85_{-0.08}^{+0.10}$ & $-21.49_{-0.02}^{+0.03}$ & $0.0042_{-0.0002}^{+0.0001}$ \\
& & & & & \\
$i^{\prime}$ & Case 1 & $-0.35_{-0.48}^{+0.43}$ & $-0.39_{-0.24}^{+0.27}$ & $-22.46_{-0.41}^{+0.44}$ & $0.0026_{-0.0004}^{+0.0006}$ \\
$i^{\prime}$ & Case 2 & $-0.38_{-0.25}^{+0.26}$ & $-0.60_{-0.16}^{+0.15}$ & $-22.45_{-0.30}^{+0.30}$ & $0.0032_{-0.0004}^{+0.0004}$ \\
$i^{\prime}$ & Case 3 & $-0.85_{-0.18}^{+0.12}$ & $-0.66_{-0.15}^{+0.08}$ & $-21.97_{-0.04}^{+0.04}$ & $0.0034_{-0.0001}^{+0.0002}$ \\
& & & & & \\
$z^{\prime}$ & Case 1 & $-0.28_{-0.58}^{+0.46}$ & $-0.42_{-0.30}^{+0.24}$ & $-22.77_{-0.45}^{+0.56}$ & $0.0027_{-0.0004}^{+0.0008}$ \\
$z^{\prime}$ & Case 2 & $-0.49_{-0.31}^{+0.29}$ & $-0.70_{-0.19}^{+0.17}$ & $-22.62_{-0.32}^{+0.38}$ & $0.0035_{-0.0006}^{+0.0006}$ \\
$z^{\prime}$ & Case 3 & $-0.81_{-0.16}^{+0.11}$ & $-0.63_{-0.12}^{+0.11}$ & $-22.22_{-0.05}^{+0.04}$ & $0.0033_{-0.0001}^{+0.0002}$ \\
\hline
\end{tabular}

$M<-19$. For the fainter part, the measured number density disagrees with Blanton et al. (2003) and Blanton et al. (2005). If we make the same comparison at $\langle z\rangle=0.65$, where the FDF covers a relatively large volume minimizing the influence of LSS, the measured LFs also follow the very local Schechter function of Blanton et al. (2005) in the faint magnitude regime. Note that Blanton et al. (2005) explicitly corrected for surface-brightness incompleteness when deriving the very local LFs.

\section{Lin et al. (1996)}

Despite the small volume covered by the FDF at low redshift, we compare in Fig. 14 (left panel) our LF with the LF derived by Lin et al. in the Las Campanas Redshift Survey (LCRS). Their sample contains 18678 sources selected from CCD photometry in a "hybrid" red Kron-Cousins $R$-band with a mean redshift of $\langle z\rangle \sim 0.1$. The solid line in Fig. 14 represents the LF in the $R$-band from Lin et al., whereas the filled circles show our $V / V_{\max }$ corrected LF derived at $0.15<z \leq 0.45$. There is rather large disagreement between the LF in the FDF and in the LCRS, which is mainly due to the different slope ( $\alpha=-0.7$ for the LCRS), but the FDF galaxy number density at the bright end also seems to be slightly higher than in the LCRS. This might be attributed partly to cosmic variance and/or to the selection method. The difference at the faint end is a well-known LCRS feature related to their selection method, which biases LCRS towards early type systems. Indeed, our LF for SED type 1 galaxies (Fig. 14) agrees very well with Lin et al.

\section{Lin et al. (1997)}

Based on 389 field galaxies from the Canadian Network for Observational Cosmology cluster redshift survey (CNOC1) selected in the Gunn-r-band, Lin et al. derived the LF in the restframe Gunn- $r$-band. In Fig. 14 (right panel) we compare our luminosity function with the LF derived by Lin et al. in the redshift range $z=0.2-0.6$. There is very good agreement between the FDF data and the CNOC1 survey concerning the LF, if we compare only the magnitude range common to both surveys. Also the slope derived in Lin et al. ( $\alpha=-1.25 \pm 0.19$, Table 2 of the paper) is compatible with the slope in the FDF.

Brown et al. (2001)

Brown et al. use $64 \mathrm{deg}^{2}$ of $V$ and $R$ images to measure the local $V$ - and $R$-band LF. They analyze about $1250 \mathrm{~V}$ and $R$ selected galaxies from the Century Survey (Geller et al. 1997) with a mean spectroscopic redshift of $\langle z\rangle \sim 0.06$.

A comparison between the LF of Brown et al. and the FDF is shown in Fig. 15 for the $V$-band (left panel) and the $R$-band (right panel). Although the agreement is quite good for the bright end, the number density of the faint-end is substantially higher in the FDF; while the slope of the LF derived in the FDF is $\alpha=-1.25$, the slope derived by Brown et al. is $\alpha=-1.09 \pm 0.09$ in the $V$ - as well as in the $R$-band.

\section{Shapley et al. (2001)}

Shapley et al. analyze 118 photometrically selected LBGs with $K_{\mathrm{s}}$-band measurements covering an area of $30 \mathrm{arcmin}^{2}$. Of these, 63 galaxies have additional $J$-band measurements and 81 are spectroscopically confirmed. Using this sample, Shapley et al. derive the luminosity function in the restframe $V$-band at redshift of $\langle z\rangle \sim$ 3.0. Figure 16 shows a comparison of the $V$-band LF derived by Shapley et al. with the LF in the FDF at $\langle z\rangle \sim 3.0$. The agreement is very good, if we again concentrate on the shaded region. On the other hand, because of the depth of the FDF we can trace the LF two magnitudes deeper and, therefore, give better constraints on the slope of the Schechter function. Comparing the faint-end of the FDF LF with the extrapolated Schechter function of Shapley et al. clearly shows that the very steep slope of $\alpha=-1.85$ is not seen in the FDF dataset.

Ilbert et al. (2005)

Ilbert et al. investigate the evolution of the galaxy LF from the VIMOS-VLT Deep Survey (VVDS) in 5 restframe bands $(U$, $B, V, R, I)$. They use about 11000 objects with spectroscopic distance information in the magnitude range $17.5 \leq I \leq 24.0$ to constrain the LF to redshift $z \sim 2$. In Fig. 17 we compare the $V, R$, and $I$ band LF of the FDF with the Schechter function derived in the VVDS survey for different redshift bins: $\quad 0.20<z \leq 0.40, \quad 0.40<z \leq 0.60, \quad 0.60<z \leq 0.80$, $0.80<z \leq 1.00, \quad 1.00<z \leq 1.30$, and $1.30<z \leq 2.00$. Because of the limited sample size of the FDF at low redshift, we could not use the same local redshift binning as Ilbert et al.. We therefore compare (Fig. 17) the VVDS Schechter function at $\langle z\rangle \sim 0.3$ and $\langle z\rangle \sim 0.5$ with the FDF LF derived at $0.2<z \leq 0.6$, as well as the Schechter function at $\langle z\rangle \sim 0.7$ and $\langle z\rangle \sim 0.9$ with the FDF LF derived at $0.6<z \leq 1.0$. There is very good agreement between the FDF data and the VVDS survey at all redshifts under investigation, if we compare only the magnitude range common to both surveys. Ilbert et al. derive the faint-end slope from shallower data, if compared with the FDF that have only a limited sensitivity for the latter. Nevertheless, in all three bands, the differences between the formal $\alpha$ derived in the FDF $\left(\alpha_{V}=-1.25 \pm 0.03\right.$ and $\alpha_{r^{\prime} \& i^{\prime}}=-1.33 \pm 0.03$ constant in redshift) and in the VVDS 

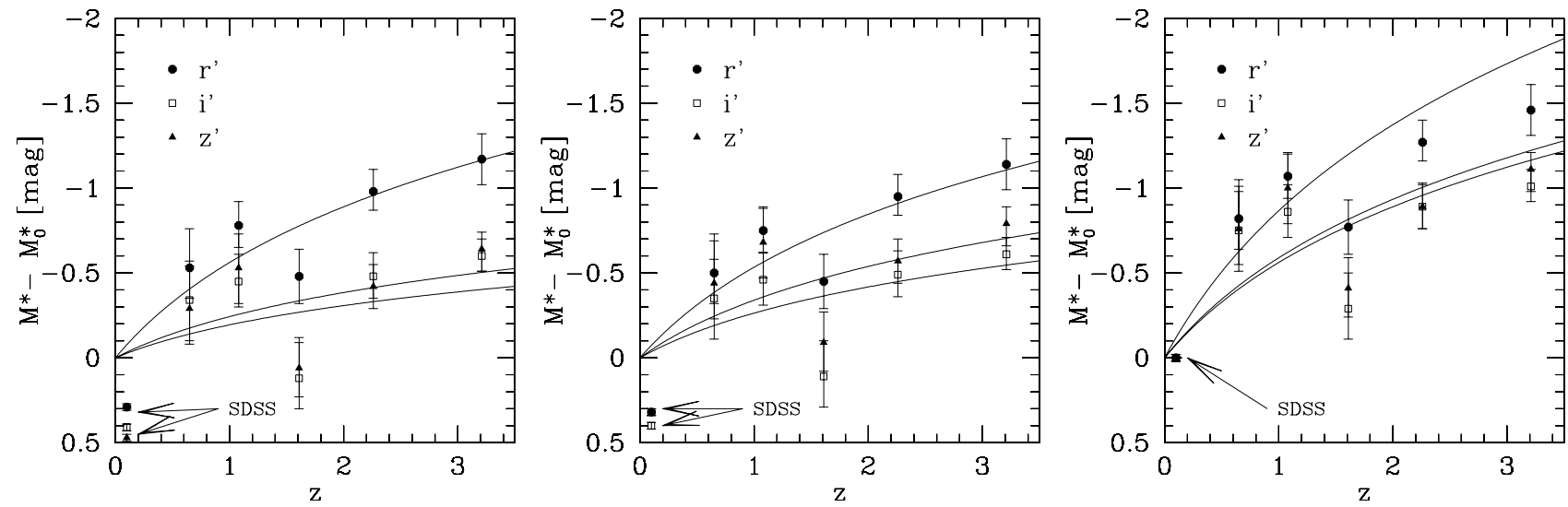

Fig. 12. Relative evolution of $M^{*}$ with redshift. The solid lines represent the best fit of the evolutionary model according to Eq. (1). Left panel: FDF LFs between redshift $\langle z\rangle \sim 0.65$ and $\langle z\rangle \sim 2.26$ are used to constrain the evolutionary model (Case 1). Middle panel: FDF LFs between redshift $\langle z\rangle \sim 0.65$ and $\langle z\rangle \sim 3.21$ are used to constrain the evolutionary model (Case 2). Right panel: FDF LFs between redshift $\langle z\rangle \sim 0.65$ and $\langle z\rangle \sim 2.26$, as well as the local LF of Blanton et al. (2003), are used to constrain the evolutionary model (Case 3) (see also text).
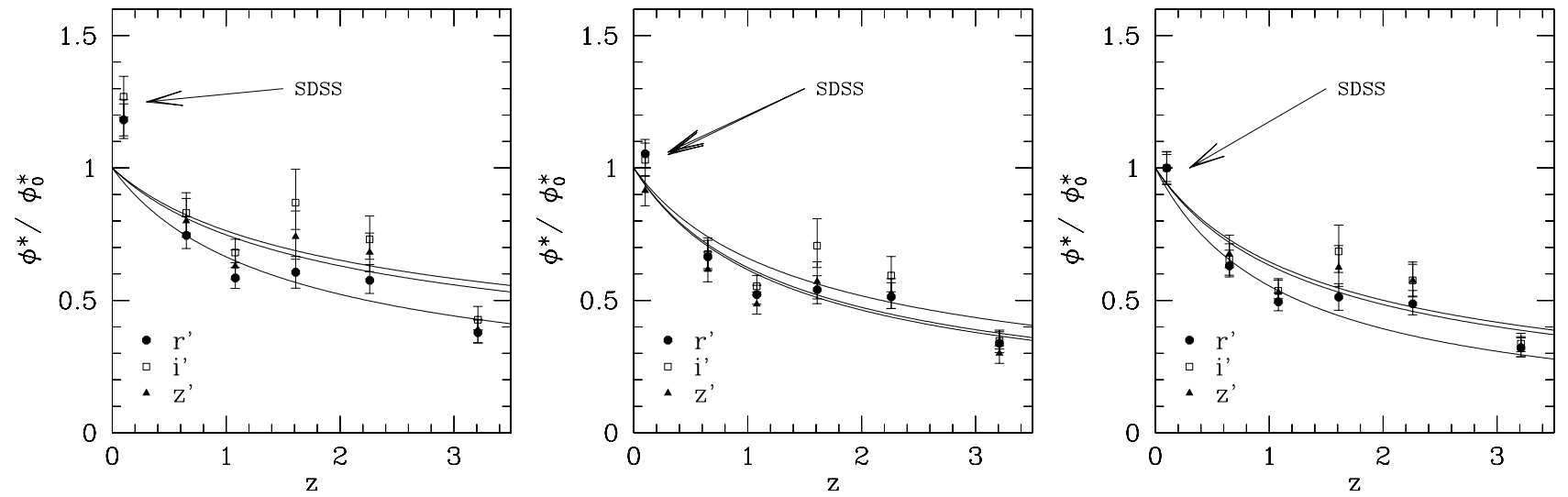

Fig. 13. Relative evolution of $\phi^{*}$ with redshift. The solid lines represent the best fit of the evolutionary model according to Eq. (1). Left panel: FDF LFs between redshift $\langle z\rangle \sim 0.65$ and $\langle z\rangle \sim 2.26$ are used to constrain the evolutionary model (Case 1). Middle panel: FDF LFs between redshift $\langle z\rangle \sim 0.65$ and $\langle z\rangle \sim 3.21$ are used to constrain the evolutionary model (Case 2). Right panel: FDF LFs between redshift $\langle z\rangle \sim 0.65$ and $\langle z\rangle \sim 2.26$, as well as the local LF of Blanton et al. (2003), are used to constrain the evolutionary model (Case 3) (see also text).

are compatible within $1 \sigma$ to $2 \sigma$ up to redshift $z \sim 0.8$ (only one bin in the $I$-band LF differs by slightly more than $2 \sigma)$. At higher redshift, we do not see the steep slope $(\sim-1.5)$ as derived by Ilbert et al. That in the FDF we are able to follow the LF about 3-4 mag deeper may explain the disagreement between the extrapolated faint-end slope of Ilbert et al. and the FDF result.

\section{Wolf et al. (2003)}

In Fig. 18 we compare the $r^{\prime}$-band LF of the FDF with the $R$ band selected luminosity function derived in the COMBO-17 survey (Wolf et al. 2003) for different redshift bins: 0.2-0.6, $0.6-0.8,0.8-1.0,1.0-1.2$. Because of the limited sample size of the FDF at low redshift we could not use the same local redshift binning as Wolf et al.. We therefore compare in Fig. 18 the COMBO17 Schechter function at $\langle z\rangle \sim 0.3$ and $\langle z\rangle \sim 0.5$ with the FDF LF derived at $0.2<z \leq 0.6$. There is very good agreement between the FDF data and the COMBO-17 survey at all redshifts under investigation, if we compare only the magnitude range common to both surveys. Although the number density of the FDF seems to be slightly higher for the restframe UV LF (FDFLF I), this is not the case if we compare the LF in the $R$-band. Wolf et al. derived the faint-end slope from relatively shallow data which have only a limited sensitivity for the latter. This may explain the disagreement between the extrapolated faint-end slope of Wolf et al. and the FDF result.

Chen et al. (2003)

The galaxy sample analyzed by Chen et al. contains $\sim 6700$ $H$-band selected galaxies (within $847 \operatorname{arcmin}^{2}$ ) in the HDFS region with complementary optical $U, B, V, R$, and $I$ colors, and $\sim 7400 \mathrm{H}$-band selected galaxies (within $561 \mathrm{arcmin}^{2}$ ) in the Chandra Deep Field South region with complementary optical $V, R, I$, and $z^{\prime}$ colors. The galaxy sample is part of the Las Campanas Infrared Survey (LCIR Marzke et al. 1999; McCarthy et al. 2001) and is based on photometric redshifts.

Figure 19 shows a comparison of the $R$-band luminosity function derived by Chen et al. with the LF in the FDF for three different redshift bins: $0.50-0.75$ (left panel), 0.75-1.00 

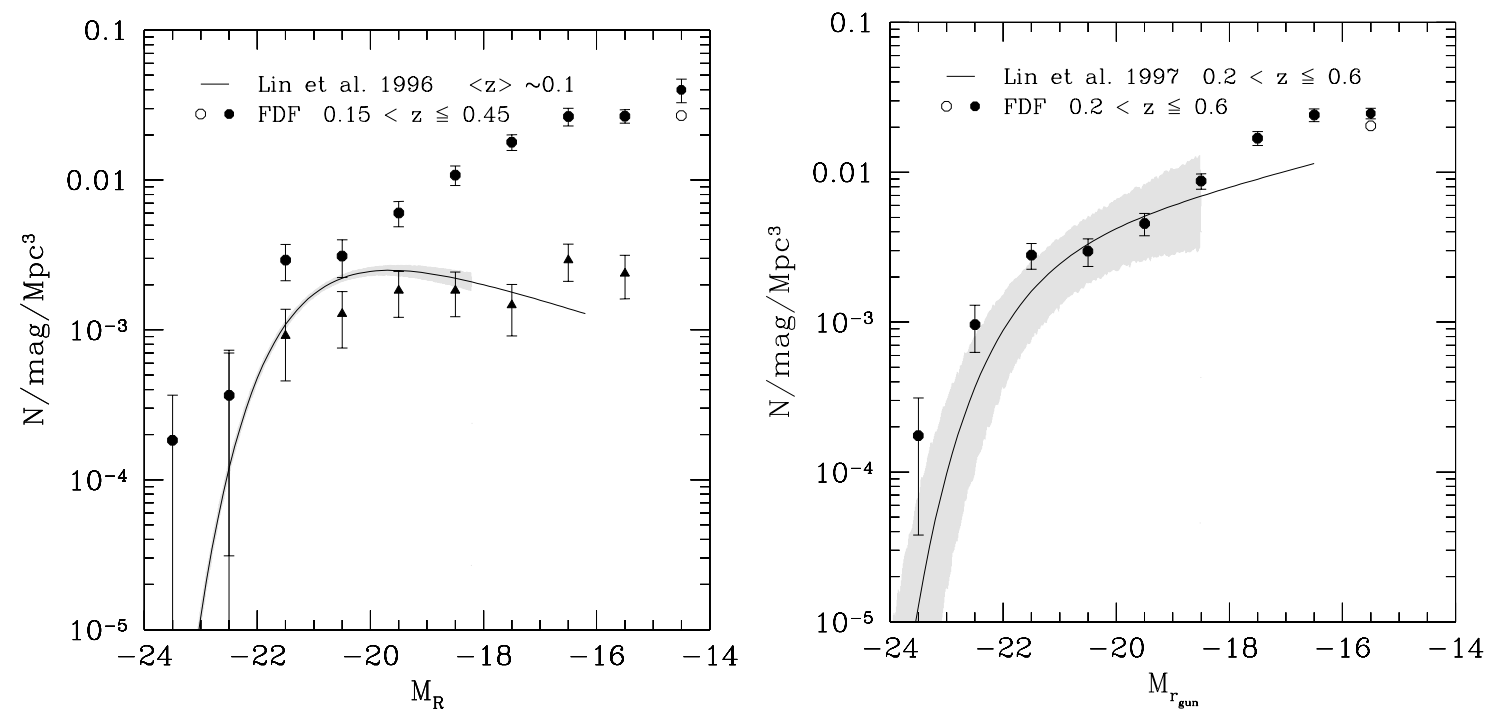

Fig. 14. Left panel: comparison of the $R$-band luminosity function of the FDF (filled/open circles $\langle z\rangle \sim 0.3$ ) with the Schechter function derived in Lin et al. (1996) $(\langle z\rangle \sim 0.1)$. The shaded region is based on $\Delta M^{*}, \Delta \phi^{*}$, and $\Delta \alpha$. The triangles show the $r^{\prime}$-band FDF LF at $\langle z\rangle \sim 0.3$ for SED type 1 galaxies. Right panel: Comparison of the Gunn- $r$-band LF of the FDF with the Schechter function derived in Lin et al. (1997) $(z=0.2-0.6)$. The shaded region is based on $\Delta M^{*}, \Delta \phi^{*}$, and $\Delta \alpha$.
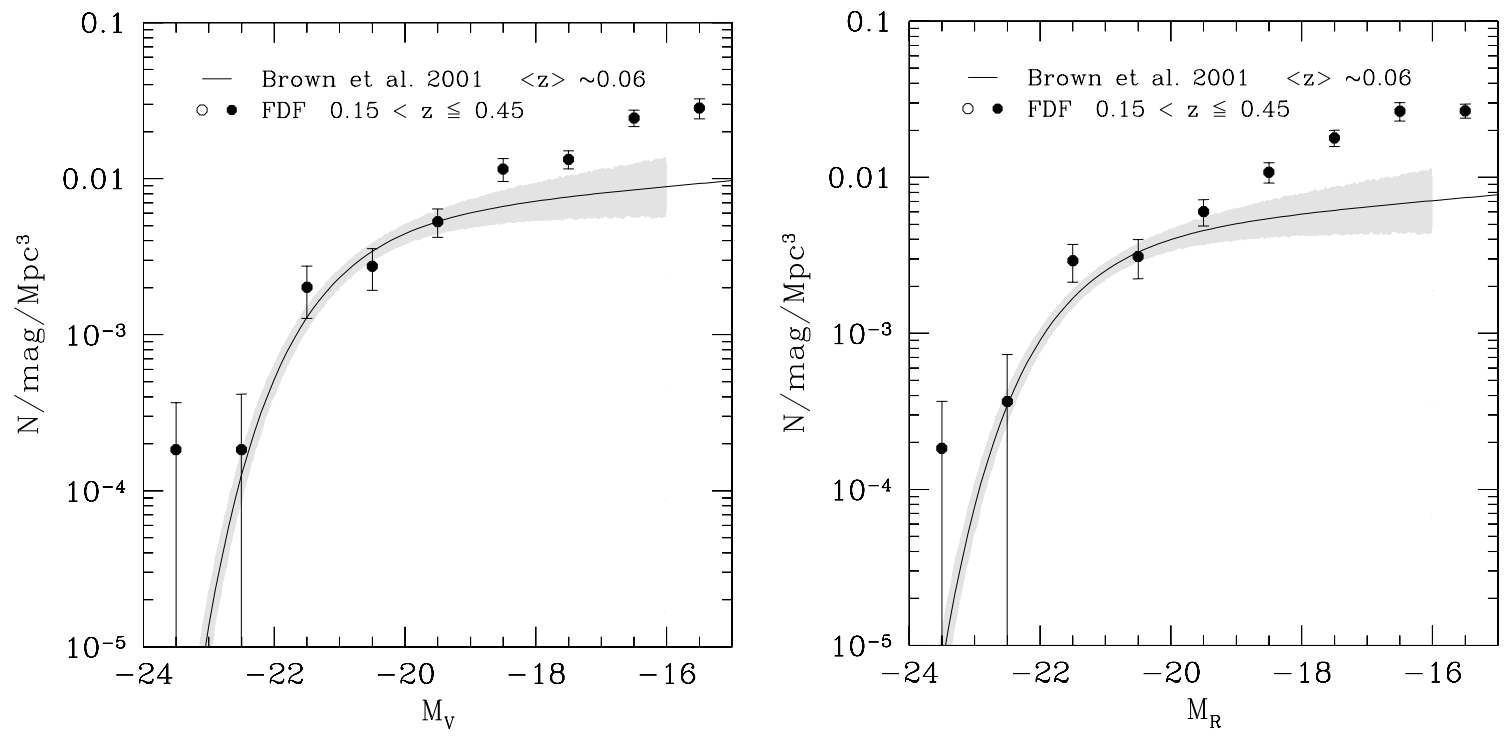

Fig. 15. Comparison of the $V$-band (left panel) and $R$-band (right panel) LF of the FDF with the local $(\langle z\rangle \sim 0.06)$ Schechter function derived in Brown et al. (2001). The shaded region is based on $\Delta M^{*}, \Delta \phi^{*}$, and $\Delta \alpha$.

(middle panel), and 1.00-1.50 (right panel). There is good agreement between the FDF LF and the Schechter function derived by Chen et al. in the lowest redshift bin $(z=0.50-0.75)$, if we compare only the magnitude range common to both surveys. At intermediate redshift $(z=0.75-1.00)$, the number density of the bright end of the FDF LF is slightly higher than in Chen et al.. On the other hand, for the highest redshift bin $(z=1.00-1.50)$ the number density of the bright end derived by Chen et al. roughly agrees with the results obtained in the FDF.

Dahlen et al. (2005)

Dahlen et al. use HST and ground-based $U$ through $K_{\mathrm{s}}$ photometry in the GOODS-S Field to measure the evolution of the $R$-band luminosity function out to $z \sim 2$. They combine a wider area, optically selected $\left(\sim 1100 \operatorname{arcmin}^{2}, R<24.5\right)$ catalog with a smaller area but deep NIR selected $\left(\sim 130 \mathrm{arcmin}^{2}, K_{\mathrm{s}}<23.2\right)$ catalog. Distances are based on photometric redshifts with an accuracy of $\Delta z /\left(z_{\text {spec }}+1\right) \sim 0.12(\sim 0.06$ after excluding $\sim 3 \%$ of outliers). To determine the restframe $R$-band galaxy luminosity function out to $z \sim 2$, they use the deep $K_{\mathrm{s}}$ selected catalog. A comparison between the $R$-band LF of Dahlen et al. and the FDF is shown in Fig. 20. There is very good agreement in nearly all redshift bins. Only at $0.82<z \leq 1.12$ and $1.37<z \leq 1.59$ does the characteristic density in the FDF seem to be slightly higher.

To summarize, we can say that the LFs derived in the FDF in general show very good agreement with other observational datasets from the literature. At the bright end of the LF, most of 


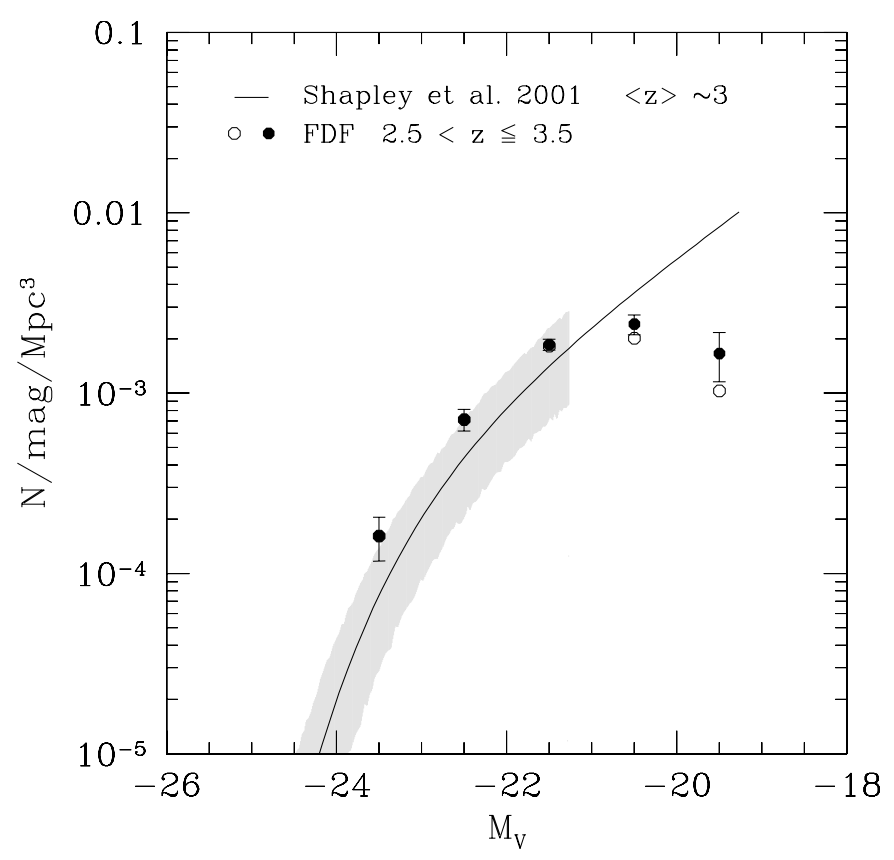

Fig. 16. Comparison of the $V$-band luminosity function of the FDF with the Schechter function derived in Shapley et al. (2001) at $\langle z\rangle \sim 3.0$. The shaded region is based on $\Delta M^{*}, \Delta \phi^{*}$, and $\Delta \alpha$, where the cut-off at low luminosity indicates the limiting magnitude of the sample.

the datasets agree within $1 \sigma$. Differences between the extrapolated Schechter function of the literature and the measured faint-end in the FDF can be attributed to the shallower limiting magnitudes of the other surveys.

\section{Comparison with model predictions}

As discussed for example in Benson et al. (2003), different physical processes are involved in shaping the bright and the faint ends of the galaxy LF. Therefore, it is interesting to compare LFs predicted by models with observational results in order to constrain those processes better. In this section we compare the $R$-band and $I$-band LFs in different redshift bins with the model predictions of Kauffmann et al. (1999).

In Fig. 21 we show the $R$-band luminosity function of the FDF, together with the semi-analytic model predictions by Kauffmann et al. (1999) ${ }^{1}$ for $\langle z\rangle \sim 0.20,\langle z\rangle \sim 0.62,\langle z\rangle \sim 1.05$, whereas in Fig. 22 we show the $I$-band LF in the redshift bins $\langle z\rangle \sim 0.20,\langle z\rangle \sim 0.62,\langle z\rangle \sim 1.05,\langle z\rangle \sim 1.46,\langle z\rangle \sim 2.12$, and $\langle z\rangle \sim 2.97$. For the $R$-band no semi-analytic model predictions are available for redshifts larger than $\langle z\rangle \sim 1.05$.

The model agrees well with the measured LFs in the $R$-band. Also for the $I$-band there is good agreement between the models and the luminosity functions derived in the FDF up to redshift $\langle z\rangle \sim 1.46$ (of course at $z \approx 0$ the model is tuned to reproduce the data). At $\langle z\rangle>1.46$ the discrepancy increases, as the model does not contain enough bright galaxies. Unfortunately, the models only predict luminosities for

\footnotetext{
1 The models were taken from: http://www.mpa-garching. mpg.de/Virgo/data_download.html
}

massive galaxies and, because of lack of resolution, do not predict galaxy number densities for faint galaxies.

\section{Summary and conclusions}

In this paper we use a sample of about 5600 I-band selected galaxies in the FORS Deep Field down to a limiting magnitude of $I=26.8 \mathrm{mag}$ to analyze the evolution of the LFs in the $r^{\prime}, i^{\prime}$, and $z^{\prime}$ bands over the redshift range $0.5<z<3.5$, thus extending the results presented in FDFLF I to longer wavelengths. All the results are based on the same catalog and the same state of the art photometric redshifts $\left(\Delta z /\left(z_{\text {spec }}+1\right) \leq 0.03\right.$ with only $\sim 1 \%$ outliers) as in FDFLF I. The error budget of the luminosity functions includes the photometric redshift error of each single galaxy, as well as the Poissonian error.

Because of the depth of the FDF, we can trace the LFs deeper than most other surveys can and therefore obtain good constraints on the faint-end slope $\alpha$ of the LF. A detailed analysis of $\alpha$ leads to similar conclusions to those found in FDFLF I for the blue regime: the faint-end of the red LFs does not show a large redshift evolution over the redshift range $0.5 \lessgtr z \lesssim 2.0$ and is compatible within $1 \sigma$ to $2 \sigma$ with a constant slope in most of the redshift bins. Moreover, the slopes in $r^{\prime}, i^{\prime}$, and $z^{\prime}$ are very similar with a best-fitting slope of $\alpha=-1.33 \pm 0.03$ for the combined bands and redshift intervals considered here.

Interestingly, an analysis of the slope of the LFs as a function of wavelength shows a prominent trend of $\alpha$ to steepen with increasing wavelength: $\alpha_{\mathrm{UV} \& u^{\prime}}=-1.07 \pm 0.04 \rightarrow$ $\alpha_{g^{\prime} \& B}=-1.25 \pm 0.03 \rightarrow \alpha_{r^{\prime} \& i^{\prime} \& z^{\prime}}=-1.33 \pm 0.03$. To better understand this wavelength dependence of the LF slope, we analyze the contribution of different galaxy types to the overall LF by subdividing our galaxy sample into 4 typical SED types with restframe $U-V$ colors between 2.3-1.9, 2.0-1.6, 1.6-0.9, and $0.9-0$ for SED type 1, 2, 3, and 4, respectively. Therefore, in a rough classification one can refer to SED types 1 and 2 (SED type 3 and 4) as red (blue) galaxies.

Although the overall LF is completely dominated in the UV regime by extreme star-forming galaxies, the overall LF in the red regime is mainly dominated by early to late type galaxies at the bright end, but extreme star-forming galaxies at the faint end. The relative contribution of the different SED type LF to the overall LF clearly changes as a function of analyzed waveband resulting (at the depth of the FDF) in a steeper slope for the overall LF in the red regime if compared to the blue regime.

To quantify the contribution of the different SED types to the total luminosity density, we derive and analyze the latter in the UV and in the red bands as a function of redshift. The contribution of type 1 and 2 galaxies to the UV LD is negligible at all analyzed redshifts, as SED types 3 and 4 completely dominate. On the other hand, the relative contribution to the overall luminosity density of type 1 and 2 galaxies is of the same order or even exceeds those of type 3 and 4 in the red bands.

We investigate the evolution of $M^{*}$ and $\phi^{*}$ (for a fixed slope $\alpha$ ) by means of the redshift parametrization introduced in FDFLF I. Based on the FDF data (Case 1 and Case 2), we find only a mild brightening of $M^{*}$ and decrease of $\phi^{*}$ with increasing redshifts in all three of these analyzed wavebands. If we follow the evolution of the characteristic luminosity from 

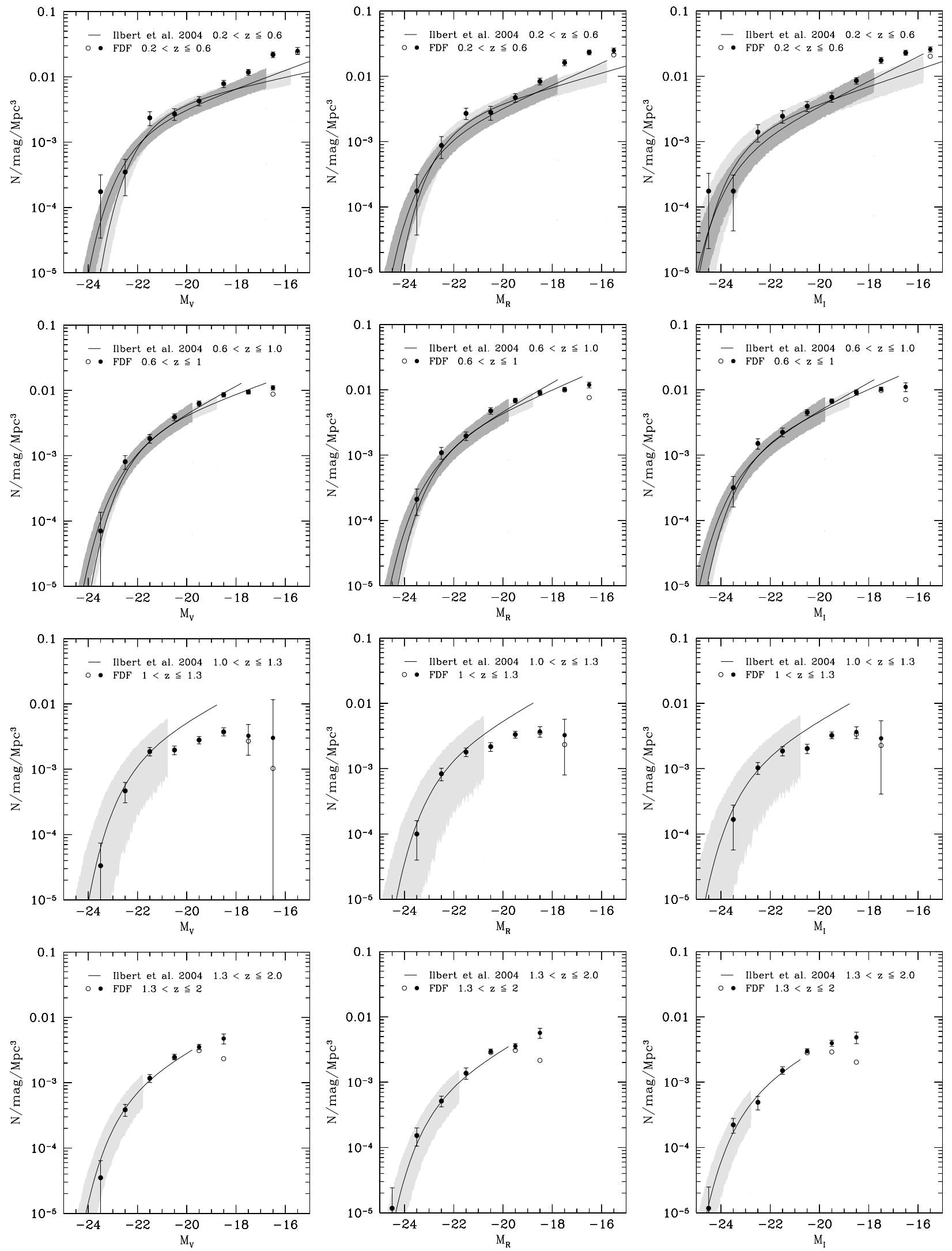

Fig. 17. Comparison of the $V$ (left panels), $R$ (middle panels), and $I$ (right panels) band LF of the FDF with the Schechter function derived in Ilbert et al. (2005) (VVDS) at $0.20<z \leq 0.40$ (first row, light gray), $0.40<z \leq 0.60$ (first row, dark gray), $0.60<z \leq 0.80$ (second row, light gray), $0.80<z \leq 1.00$ (second row, dark gray), $1.00<z \leq 1.30$ (third row), and $1.30<z \leq 2.00$ (fourth row). The shaded regions of all plots with $z \leq 1$ are based on $\Delta M^{*}, \Delta \phi^{*}$, and $\Delta \alpha$. Only in the two high redshift bins (third and fourth row) is the shaded region based on $\Delta M^{*}$ and $\Delta \phi^{*}$. Please note that we use the average error, if the upper and lower values reported by Ilbert et al. (2005) disagree. 

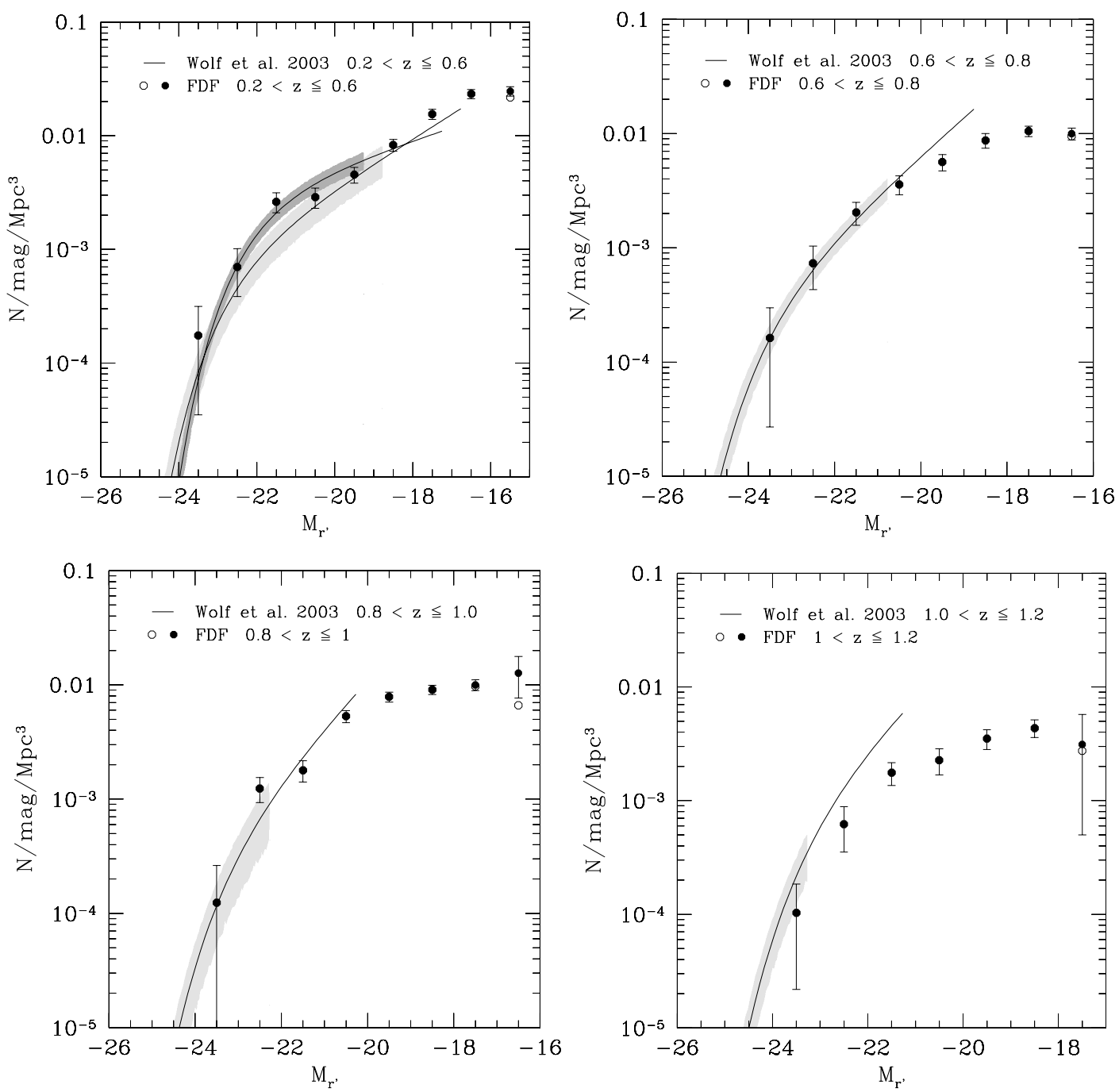

Fig. 18. Comparison of the LF in the $r^{\prime}$-band of the FDF with the Schechter function derived in Wolf et al. $(2003$ ): $0.2<z \leq 0.4$ (upper left panel, light gray), $0.4<z \leq 0.6$ (upper left panel, dark grey), $0.6<z \leq 0.8$ (upper right panel), $0.8<z \leq 1.0$ (lower left panel), $1.0<z \leq 1.2$ (lower right panel). The shaded regions of nearly all plots are based on $\Delta M^{*}, \Delta \phi^{*}$, and $\Delta \alpha$. Only in the highest redshift bin (lower right panel) the shaded region is based only on $\Delta M^{*}$ and $\Delta \phi^{*}$. The cut-off at low luminosity indicates the limiting magnitude of the sample.
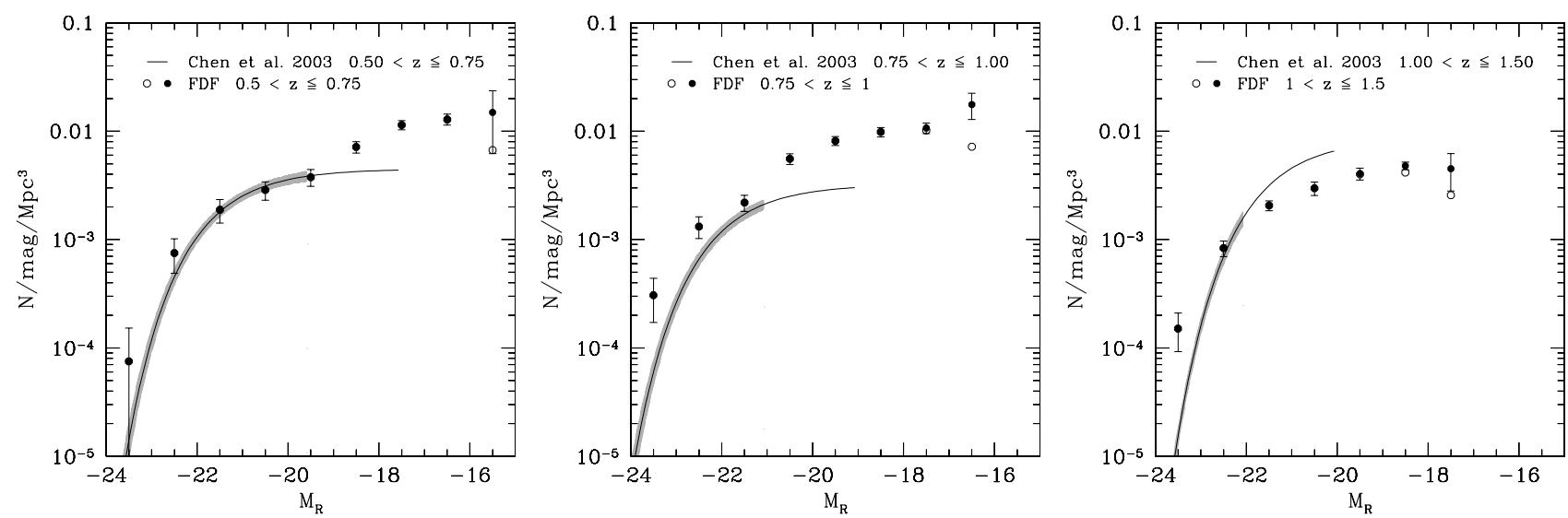

Fig. 19. Comparison of the luminosity function in the $R$-band of the FDF with the Schechter function derived in Chen et al. (2003): $0.50<z \leq 0.75$ (left panel), $0.75<z \leq 1.00$ (middle panel), and $1.00<z \leq 1.50$ (right panel). The shaded region is based on $\Delta M^{*}, \Delta \phi^{*}$ and $\Delta \alpha$ for $0.50<z \leq 0.75$ (left panel). For $0.75<z \leq 1.00$ (middle panel) and $1.00<z \leq 1.50$ (right panel), the shaded region is based only on $\Delta M^{*}$ and $\Delta \phi^{*}$. The cut-off at low luminosity indicates the limiting magnitude of the sample. 

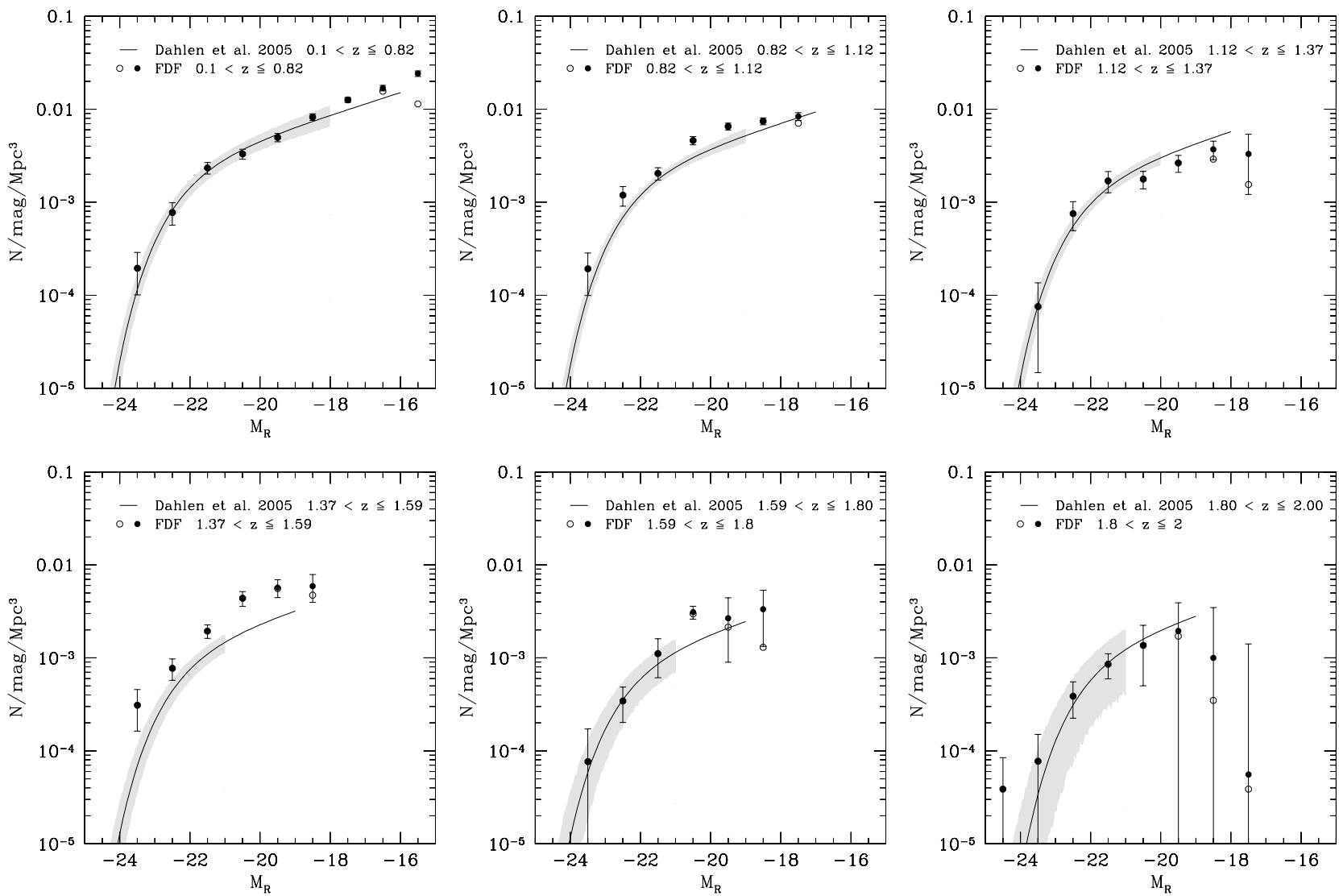

Fig. 20. Comparison of the $R$-band LF of the FDF with the Schechter function derived in Dahlen et al. (2005) at $0.10<z \leq 0.82$, $0.82<z \leq 1.12,1.12<z \leq 1.37,1.37<z \leq 1.59,1.59<z \leq 1.80$, and $1.80<z \leq 2.00$ (from upper left to lower right panel). The shaded region is based on $\Delta M^{*}, \Delta \phi^{*}$, and $\Delta \alpha$ for all panels. The cut-off of the shaded region at low luminosity indicates the limiting magnitude of the sample.
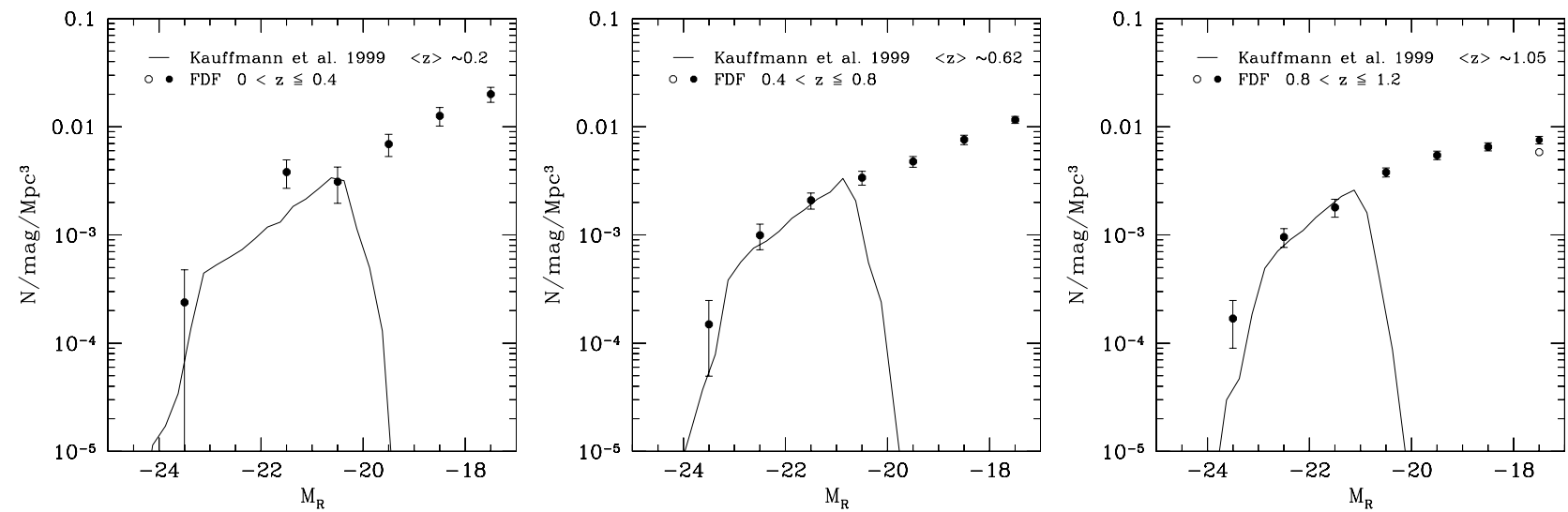

Fig. 21. Comparison of the $R$-band LF of the FDF with predictions based on Kauffmann et al. (1999) (solid line): $\langle z\rangle \sim 0.20,\langle z\rangle \sim 0.62$, and $\langle z\rangle \sim 1.05$, (from left to right panel). The filled (open) symbols show the LF corrected (uncorrected) for $V / V_{\max }$. The drops of the theoretical curves towards the faint ends are caused by the limited mass resolution of the models, see Kauffmann et al. (1999) for details.

$\langle z\rangle \sim 0.5$ to $\langle z\rangle \sim 3$, we find an increase of $\sim 0.8$ mag in the $r^{\prime}$, and $\sim 0.4 \mathrm{mag}$ in the $i^{\prime}$ and $z^{\prime}$ bands. Simultaneously the characteristic density decreases by about $40 \%$ in all the analyzed wavebands. We compare the LFs with previous observational datasets and discuss discrepancies. As for the blue bands, we find good/very good agreement with most of the datasets, especially at the bright end. Differences in the faint-end slope in most cases can be attributed to the shallower limiting magnitudes of the other surveys.

We also compare our results with predictions of semianalytical models at various redshifts. The semi-analytical models predict LFs that describe the data at low redshift very well, but as for the blue bands, they show growing disagreement with increasing redshifts. Unfortunately, the models only 

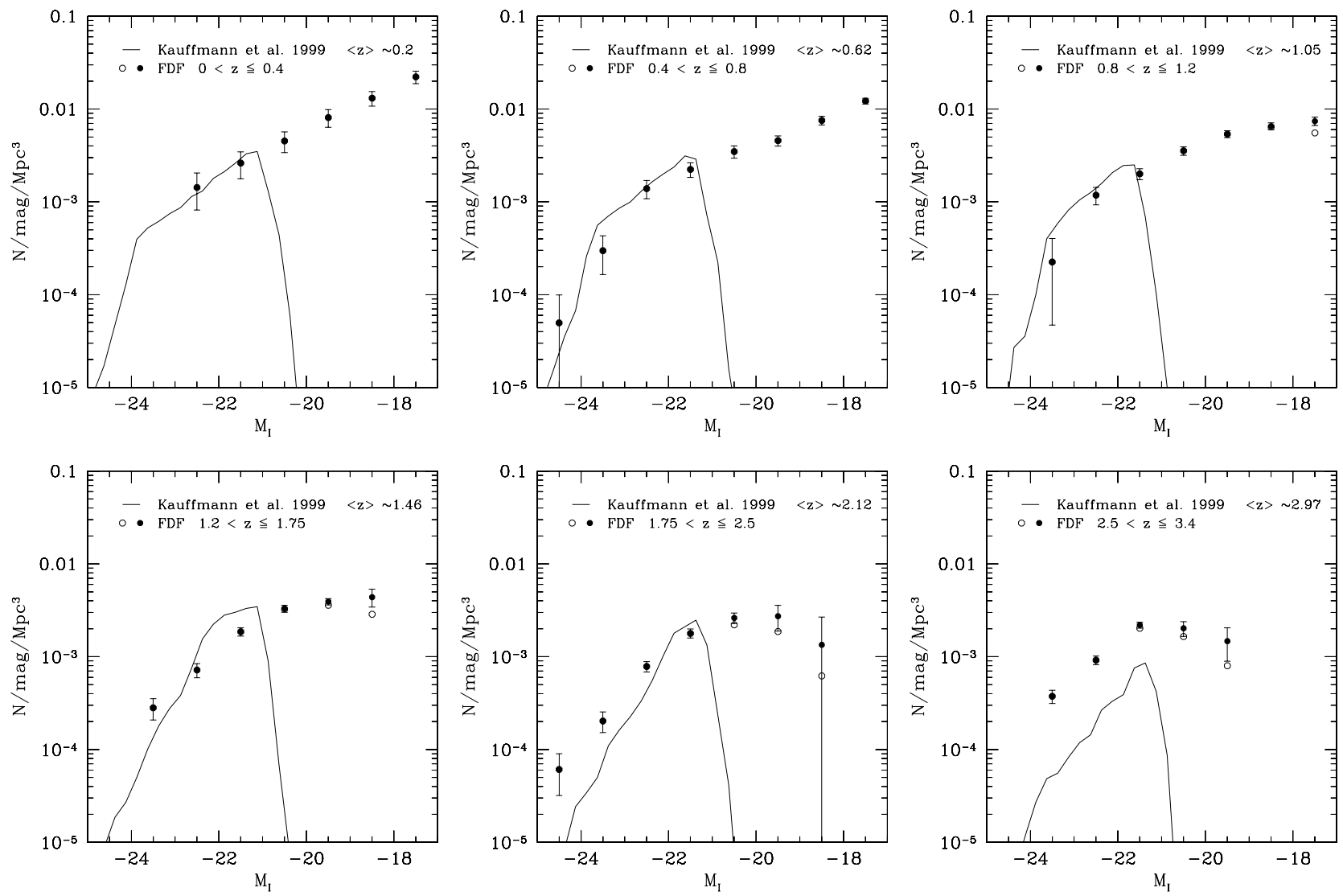

Fig. 22. Comparison of the $I$-band LF of the FDF with predictions based on Kauffmann et al. (1999) (solid line): $\langle z\rangle \sim 0.20,\langle z\rangle \sim 0.62$, $\langle z\rangle \sim 1.05,\langle z\rangle \sim 1.46,\langle z\rangle \sim 2.12$, and $\langle z\rangle \sim 2.97$ (from upper left to lower right panel). The filled (open) symbols show the LF corrected (uncorrected) for $V / V_{\max }$. The drops of the theoretical curves towards the faint ends are caused by the limited mass resolution of the models; see Kauffmann et al. (1999) for details.

predict luminosities for massive galaxies,so that a comparison between the predicted and observed galaxy number densities for low luminosity galaxies $\left(L \lesssim L^{*}\right)$ could not be done.

Acknowledgements. We thank the anonymous referee for the helpful comments that improved the presentation of the paper. We acknowledge the support of the ESO Paranal staff during several observing runs. This work was supported by the Deutsche Forschungsgemeinschaft, DFG, SFB 375 Astroteilchenphysik, SFB 439 (Galaxies in the young Universe), the Volkswagen Foundation (I/76520), and the Deutsches Zentrum für Luft- und Raumfahrt (50 OR 0301).

\section{References}

Andreon, S. 2004, A\&A, 416, 865

Arnouts, S., Schiminovich, D., Ilbert, O., et al. 2005, ApJ, 619, L43

Böhm, A., Ziegler, B. L., Saglia, R. P., et al. 2004, A\&A, 420, 97

Baldry, I. K., Glazebrook, K., Budavári, T., et al. 2005, MNRAS, 358, 441

Balogh, M. L., Christlein, D., Zabludoff, A. I., \& Zaritsky, D. 2001, ApJ, 557, 117

Bender, R., Appenzeller, I., Böhm, A., et al. 2001, in ESO/ECF/STScI Workshop on Deep Fields, ed. S. Christiani (Berlin: Springer), 96

Benson, A. J., Bower, R. G., Frenk, C. S., et al. 2003, ApJ, 599, 38

Blanton, M. R., Dalcanton, J., Eisenstein, D., et al. 2001, AJ, 121, 2358
Blanton, M. R., Hogg, D. W., Bahcall, N. A., et al. 2003, ApJ, 592, 819

Blanton, M. R., Lupton, R. H., Schlegel, D. J., et al. 2005, ApJ, 631, 208

Brown, W. R., Geller, M. J., Fabricant, D. G., \& Kurtz, M. J. 2001, AJ, 122, 714

Budavári, T., Szalay, A. S., Charlot, S., et al. 2005, ApJ, 619, L31

Chen, H., Marzke, R. O., McCarthy, P. J., et al. 2003, ApJ, 586, 745

Cole, S., Norberg, P., Baugh, C. M., et al. 2001, MNRAS, 326, 255

Croton, D. J., Farrar, G. R., Norberg, P., et al. 2005, MNRAS, 356, 1155

Dahlen, T., Mobasher, B., Somerville, R. S., et al. 2005, ApJ, 631, 126

Driver, S. P., Liske, J., Cross, N. J. G., De Propris, R., \& Allen, P. D. 2005, MNRAS, 360, 81

Drory, N., Bender, R., Feulner, G., et al. 2003, ApJ, 595, 698

Drory, N., Salvato, M., Gabasch, A., et al. 2005, ApJ, 619, L131

Feulner, G., Bender, R., Drory, N., et al. 2003, MNRAS, 342, 605

Feulner, G., Gabasch, A., Salvato, M., et al. 2005, ApJ, 633, L9

Gabasch, A., Bender, R., Seitz, S., et al. 2004a, A\&A, 421, 41 (FDFLF I)

Gabasch, A., Salvato, M., Saglia, R. P., et al. 2004b, ApJ, 616, L83

Geller, M. J., Kurtz, M. J., Wegner, G., et al. 1997, AJ, 114, 2205

Giallongo, E., Salimbeni, S., Menci, N., et al. 2005, ApJ, 622, 116

Heidt, J., Appenzeller, I., Gabasch, A., et al. 2003, A\&A, 398, 49

Huang, J.-S., Glazebrook, K., Cowie, L. L., \& Tinney, C. 2003, ApJ, 584,203

Hunter, D. A., Gallagher, J. S., \& Rautenkranz, D. 1982, ApJS, 49, 53 
Ilbert, O., Tresse, L., Zucca, E., et al. 2005, A\&A, 439, 863

Iwata, I., Ohta, K., Tamura, N., et al. 2003, PASJ, 55, 415

Kauffmann, G., Colberg, J. M., Diaferio, A., \& White, S. D. M. 1999, MNRAS, 303, 188

Kochanek, C. S., Pahre, M. A., Falco, E. E., et al. 2001, ApJ, 560, 566 Lin, H., Kirshner, R. P., Shectman, S. A., et al. 1996, ApJ, 464, 60

Lin, H., Yee, H. K. C., Carlberg, R. G., \& Ellingson, E. 1997, ApJ, 475,494

Liske, J., Lemon, D. J., Driver, S. P., Cross, N. J. G., \& Couch, W. J. 2003, MNRAS, 344, 307

Loveday, J. 2000, MNRAS, 312, 557

Madau, P., Ferguson, H. C., Dickinson, M. E., et al. 1996, MNRAS, 283, 1388

Madau, P., Pozzetti, L., \& Dickinson, M. 1998, ApJ, 498, 106

Marzke, R., McCarthy, P. J., Persson, E., et al. 1999, in Photometric Redshifts and the Detection of High Redshift Galaxies, ASP Conf. Ser., 191, 148

McCarthy, P. J., Carlberg, R. G., Chen, H.-W., et al. 2001, ApJ, 560 , L131

Noll, S., Mehlert, D., Appenzeller, I., et al. 2004, A\&A, 418, 885
Norberg, P., Cole, S., Baugh, C. M., et al. 2002, MNRAS, 336, 907

Ouchi, M., Shimasaku, K., Okamura, S., et al. 2004, ApJ, 611, 660

Pérez-González, P. G., Rieke, G. H., Egami, E., et al. 2005, ApJ, 630, 82

Pannella, M., Hopp, U., Saglia, R., et al. 2005, ApJL, submitted Poli, F., Menci, N., Giallongo, E., et al. 2001, ApJ, 551, L45

Popesso, P., Biviano, A., Böhringer, H., \& Romaniello, M. 2005, A\&A, in press [arXiv: astro-ph/0506201]

Pozzetti, L., Cimatti, A., Zamorani, G., et al. 2003, A\&A, 402, 837

Schechter, P. 1976, ApJ, 203, 297

Schmidt, M. 1968, ApJ, 151, 393

Shapley, A. E., Steidel, C. C., Adelberger, K. L., et al. 2001, ApJ, 562, 95

Steidel, C. C., Adelberger, K. L., Giavalisco, M., Dickinson, M., \& Pettini, M. 1999, ApJ, 519, 1

Tinsley, B. M. 1971, A\&A, 15, 403

Trentham, N., Sampson, L., \& Banerji, M. 2005, MNRAS, 357, 783

Treyer, M., Wyder, T. K., Schiminovich, D., et al. 2005, ApJ, 619, L19

Wolf, C., Meisenheimer, K., Rix, H.-W., et al. 2003, A\&A, 401, 73 